\title{
Experimental Study of Wake Characteristics in Tidal
}

\section{Turbine Arrays}

M. Nuernberg, L.Tao

Department of Naval Architecture, Ocean and Marine Engineering, University of Strathclyde, Glasgow G4 OLZ, UK 


\section{Abstract}

2 For the successful deployment of large scale tidal turbine arrays occupying a large part of tidal

3 channels, understanding the effects of wake interaction in densely spaced arrays is of

4 importance. A comprehensive set of experiments has been conducted with scaled tidal turbines

5 to investigate the resulting wake characteristics in a number of different staggered array

6 configurations with up to four turbines on a designated support frame. Wake velocity deficits

7 and turbulence intensities at a number of locations within and downstream of the array are

8 presented and in addition the flow field recordings from Particle Image Velocimetry (PIV)

9 measurements are presented for visual investigation of the resulting wake field and wake

10 characteristics along the array centre line. The experiments show that lateral and longitudinal

11 spacing variations of the individual devices vary the resulting flow field downstream of the

12 array section significantly. Lateral spacing can be optimized to result in beneficial flow effects

13 that accelerate the downstream wake recovery. Very close spacing however leads to

14 significantly reduced velocity recovery. Longitudinal spacing shows less significant influence,

15 especially for configurations with wide lateral distances. Differences in wake velocity deficit

16 of up to $10 \%$ have been identified and suggest array wake recovery in and downstream of

17 staggered sections, in areas of lower ambient turbulence levels, to be more significantly

18 influenced by the lateral spacing especially towards the front rows of the array. With every

19 additional array section the increasing turbulence intensity within the array is anticipated to

20 reduce this effect.

\section{Keywords:}

22 Tidal turbine arrays; wake measurements; turbulence intensity; Particle Image Velocimetry (PIV)

*Author for correspondence (longbin.tao@strath.ac.uk)

$\dagger$ Present address: Department of Naval Architecture, Ocean and Marine Engineering, University of Strathclyde, Glashow G4 OLZ, UK 


\section{$1 \quad 1$. Introduction}

21.1 Marine \& Renewable Energy

3 Continuing efforts are being made to achieve the challenging 2020 renewable energy targets

4 by providing a diverse, low-carbon and secure energy mix [1]. Within the field of renewable

5 electricity, the oceans as a direct source of sustainable energy, predominantly through

6 conversion of kinetic energy in waves and tidal currents have gained considerable interest for

7 the generation of electricity over the past and have an estimated combined potential of 8 providing up to $20 \%$ of the UK's electricity demand [2].

9 Electricity generation from tidal currents draws upon similarities with the wind energy sector,

10 however the challenges posed to make this electricity economically viable are more complex

11 due to operating in highly temporarily and spatially varying flows and across a large part of the current boundary layer. Additionally, the complex bathymetry and influences of waves and sediment transport affect the performance and wake evolution of tidal turbines. Tidal stream energy has the advantages of high energy density, being practically out of sight and being the most reliable and long term predictable renewable energy source available.

16 Currently, the industry is preparing to move from single demonstrator projects to first generation commercial arrays with installation and first stage operation currently in progress at the MeyGen project site (MeyGen confirmed first power of single device in 2016 [3-5] and has since installed 4 turbines, generating electricity [6]). Further, and accelerated development

20 of large scale tidal turbine arrays is anticipated beyond 2020 [1].

21 Due to the high cost involved and complexity of operating in harsh environments, improved understanding and prediction of likely energy yields is needed which requires comprehensive studies of the complex flow interactions and the effect on tidal turbines when arranged in large arrays. Experimental studies that investigate the flow characteristics within model scale tidal 
1 turbine arrays are a vital step towards validation and application of numerical models for

2 further investigation of array deployment at reduced cost and risks.

31.2 Problem and Inadequacies of current status

4 The design and performance of individual devices has been investigated using a range of

5 numerical simulations, some of which in combination with experiments [7], and experimental

6 studies ranging from small actuator disks [8] through scaled tidal turbines to large scale

7 prototypes deployed in open water [9]. The assessment of tidal turbines operating in array has

8 largely been done numerically using simplified Blade Element Momentum theory (BEMT)

9 through fully resolved three dimensional computational fluid dynamics (CFD) to large scale

10 hydro-environmental modelling [10-13] examining the performance and energy yields of

11 single devices and proposed arrays without experiments to support the outcome of numerical

12 calculations. Theoretical models have shown that power generation of turbine operating in tidal

13 channel is improved by increasing the blockage ratio [14] and where it's practically infeasible

14 to occupy the entire width of a channel, the energy produced reduces from $1 / 3$ of the tidal fence

15 for turbines occupying a large fraction of the channel cross-section to $2 / 3$ of that of a tidal fence

16 across the entire cross section for turbines covering a small section of the channel cross

17 sections. Extending this to tidal turbine arrays has shown that with increasing local blockage

18 efficiency is increased up to a level where total array efficiency is reduced due to array scale

19 chocking effects reducing the flow through the entire array [15]. Investigations of centred and

staggered arrays using theoretical linear momentum actuator disk theory [16] showed that for

21 multi row arrays the device spacing within each row is a function of the number of rows,

22 reducing the optimum blockage from 0.4 for one fence to 0.12 for two fences with a distance

23 downstream between both of the order of the fence width. With additional rows this trend

24 continues, thus recommendations for large spacing within each row to maximize power per

25 turbine similar to offshore wind farms are made. 
1 Near wake characterisation up to 10 diameter (D) downstream of a single turbine by experiment

2 has been presented previously with focus on ambient turbulence [17] and device generated

3 turbulence [18] affecting the wake structure. Detailed accounts have been taken of the

4 evolution of wake velocity and turbulence at a number of positions throughout the wakes,

5 showing the recovery towards ambient conditions at a number of different operating conditions

6 and highlighting the accelerated wake recovery with increased ambient turbulence levels.

7 Three dimensional volumetric flow field visualisation of the wake of a miniature tidal turbine

8 have been presented by Chamorro et al. [19] up to 5D behind the turbine. The small-scale wake

9 measurements revealed detailed information about dynamic wake features such as the wake

10 expansion being proportional to one-third-power law. The dynamics interaction of vortices and

11 their breakdown to the loss of wake stability occurring within 1D downstream of the turbine.

12 Recent experimental studies that investigated wake characteristics and interaction effects of

13 multiple tidal turbines operating in in-line configuration and first generation arrays are still

14 limited in number and complexity of formations tested [20-23] and focused mainly on the

15 interaction effects for different downstream spacings. Comparison of wake characteristics of a

16 single device and a second device operating within the wake of an upstream device [20] showed

17 similar behaviour for the downstream wake as compared to the high ambient turbulence case

18 for a single turbine. Performance of second row devices with spacings between $2 \mathrm{D}$ and $12 \mathrm{D}$ in

19 low ambient turbulence environments was about half of the upstream turbine. For higher

20 turbulent intensities, the performance was within $10 \%$ for downstream placing of $6 \mathrm{D}$ and more.

21 Flow measurements in these experiments were primarily done using pointwise Acoustic

22 Doppler Velocimetry (ADV) or Laser Doppler velocimetry (LDV) measurements across the

23 wake region for wake characterisation and performance evaluation.

24 Particle Image Velocimetry (PIV) has so far only been applied to a very limited number of tidal turbine studies and their wake characterisation. While Shi, Atlar and Norman [24] investigated 
1 the blade scale flow using PIV, wake characterisation using PIV measurements by Good,

2 Hamill, Whittaker and Robinson [25] was shown to investigate the flow field around a tidal

3 turbine structure in an experimental test channel to account for the change in flow field due to

4 the support structure and a rotor spinning freely. Flow acceleration on top and bottom tip of

5 the device have been observed and the vertical profiles show the characteristic transition from

6 hat shaped to bell shaped profile between 0.5D and 1D. No power take-off was simulated and

7 measurements extended downstream only 1.5D.

8 Myers and Bahaj [21] used up to three actuator disks to investigate wake interaction with

9 varying lateral separation between two disks in a single row array, and a two-row array with a

10 third actuator disk being located 3D downstream of the first row. Optimum lateral spacing was

11 shown to increase flow velocity between adjacent disks with $1.5 \mathrm{D}$ spacing, where smaller

12 spacing showed combined wakes of each individual disk and increased thrust coefficients. By

13 placing a third disk in the accelerated jet between the two upstream disks, the near wake deficit

14 of the first row turbines was reduced as these were diverted around the downstream disk. The

15 combined wakes further downstream of the array showed increased width and higher centre

16 line velocity deficits due to reduced mixing with ambient flow and penetrating towards the

17 wake centreline. No increase in thrust of the upstream disks was recorded when placing a third

18 disk downstream and the thrust coefficient of the downstream disk is equivalent to the upstream

19 disk with $C_{T} \cong 0.92$, using the inflow velocity at the position of the disk in its absence.

20 Previous experiments by Myers and Bahaj [26] investigated the effects of boundary proximity

21 by altering the vertical position of actuator disks in an experimental flume and have shown

22 increased wake velocity deficit with increasing proximity to the sea bed. Tests with increased

23 seabed roughness representing more accurately the realistic ambient turbulence levels are used

24 to investigate accelerated wake re-energisation and have shown merging of the disk and seabed wake for close proximity to the seabed thus extending the wake further downstream. This is an 
1 important consideration for the design of support structure and the authors showed that

2 increased turbulence intensities alone, especially at low current velocities may not translate

3 into accelerated wake recovery as expected.

4 A number of model scale arrays has been tested in a wide flume [22] to investigate changes in

5 wake structure when arranging turbines in array. Longitudinal, lateral and vertical velocity

6 profiles and turbulence intensities are presented for several arrangements of multiple turbines

7 located in a single row. For lateral separation of 2D and less, wake merging between adjacent

8 wakes was observed resulting in nearly constant velocity deficit across the wake width by 8D

9 downstream. For these configurations, thinner wake shapes and faster wake recovery are seen

10 in areas between wakes due to increased turbulence levels. The effect of multiple rows on the

11 wake profiles at two distances downstream of the group of rotors and thrust coefficients for

12 individual rotors has been presented by Olczak, Stallard, Feng and Stansby [23] showing

13 reducing thrust coefficients for each subsequent row of turbines and in comparison with RANS-

14 BEMT simulations decreasing accuracy of the predicted values with each additional row

15 downstream. especially for turbines in the centre of the array. The wake velocity deficit was

16 largest for the outer turbines and reduced, but showed individual peaks for each rotor within

$172 \mathrm{D}$ and $4 \mathrm{D}$ of the last row.

18 To the author's knowledge, this study is the first experimental study investigating wake effects

19 in second generation tidal turbine arrays using PIV measurements of the resulting flow field 20 within and downstream of the array section. The study involves a number of array 21 configurations with up to four small scale (1/70th) turbines placed on a dedicated support frame 22 on the bottom section of a large circulating water channel. Various array configurations of 23 lateral and longitudinal distances are tested by changing the location of the second row of 24 turbines and using three different lateral distances between the turbines on the second row. The wake characteristics with turbines located either side of the array centre line are investigated. 
1 Due to the characteristics of the facility and set-up of measurement equipment, non- intrusive

2 PIV measurements present a vertical section of the resulting flow field in the wake of single,

3 three and four turbine array arrangements along the array centreline. No measurements were

4 taken to investigate the lateral expansion of the wake. The main objective is to determine trends

5 arising from changing spacing configurations such as flow acceleration and accelerated wake

6 recovery due to increased turbulence levels to gain insight into the resulting flow field and the

7 possible spacing of such array sections.

\section{2. Experimental Approach}

9 All array tests were conducted in the Circulating Water Channel (CWC) at Shanghai Jiao Tong

10 University (SJTU) with a working section of $8 \mathrm{~m}$ length, $3 \mathrm{~m}$ width and $1.6 \mathrm{~m}$ water depth.

11 Current velocities ranged from $0.25 \mathrm{~m} / \mathrm{s}$ to $0.8 \mathrm{~m} / \mathrm{s}$ with three different velocities being presented

12 here, $0.25 \mathrm{~m} / \mathrm{s}, 0.44 \mathrm{~m} / \mathrm{s}$ and $0.8 \mathrm{~m} / \mathrm{s}$. The free stream ambient current velocity has low turbulence

13 intensity of $2 \%$ based on the specification of test facility and PIV measurements. Undisturbed

14 flow measurements have been taken at several positions through the test-section to confirm

15 ambient current and turbulence conditions prior to conducting experiments. The vertical

16 profiles of the ambient flow in Fig. 1 show small velocity variations across the lower part of

17 the rotor and constant velocities above the rotor hub height. Turbulence intensity (I) is

18 calculated based on the root mean square value of the velocity fluctuations and the time

19 averaged ambient flow velocity. The turbulence intensity is calculated as shown in (1) and

20 velocity deficit in (2) as:

Turbulence Intensity

$$
I=\frac{\sqrt{\frac{1}{3}\left(u^{\prime 2}+v^{\prime 2}+w^{\prime 2}\right)}}{U_{0}}
$$

Velocity Deficit

where $u^{\prime}, v^{\prime}$ and $w^{\prime}$ are the root mean square values of the turbulence velocity fluctuations. $U_{\mathrm{x}}$ is the time averaged in-stream velocity component and $U_{0}$ the ambient flow velocity. 


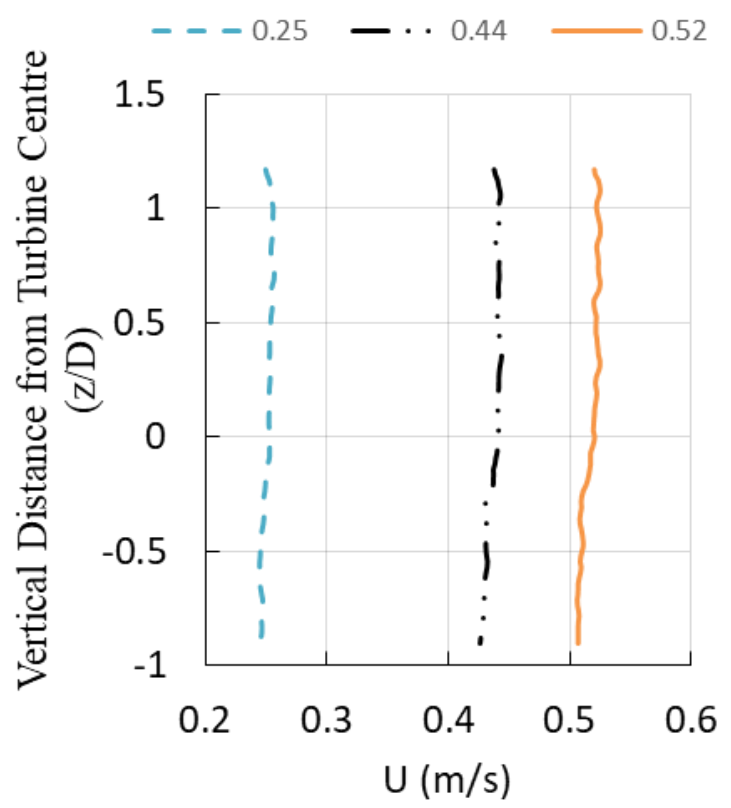

2 Fig. 1 - Vertical Current Profile in the CWC for $0.25 \mathrm{~m} / \mathrm{s}$ (Dashed), 0.44m/s (Dash Dot) and $3 \quad 0.52 \mathrm{~m} / \mathrm{s}$ (Solid)

4 The experimental arrangement taken as a section of a staggered tidal turbine array of four 5 turbines is highlighted by the box in Fig. 2(a) from Mycek, Gaurier, Germain, Pinon and 6 Rivoalen [20]. For investigation of the initial array effects, the focus will be the centre line 7 array wake characterisation across a number of device spacings in longitudinal (constant a1 8 with varying a3) and lateral (variation of b1) direction. Distances are given in terms of 9 downstream distance from the first rotor divided by the turbine diameter (D).

10 The measurements thus cover the downstream distance ranging from $2 \mathrm{D}-20 \mathrm{D}$ on the array 11 centre line as shown in Fig. 2 (b). 
(a)

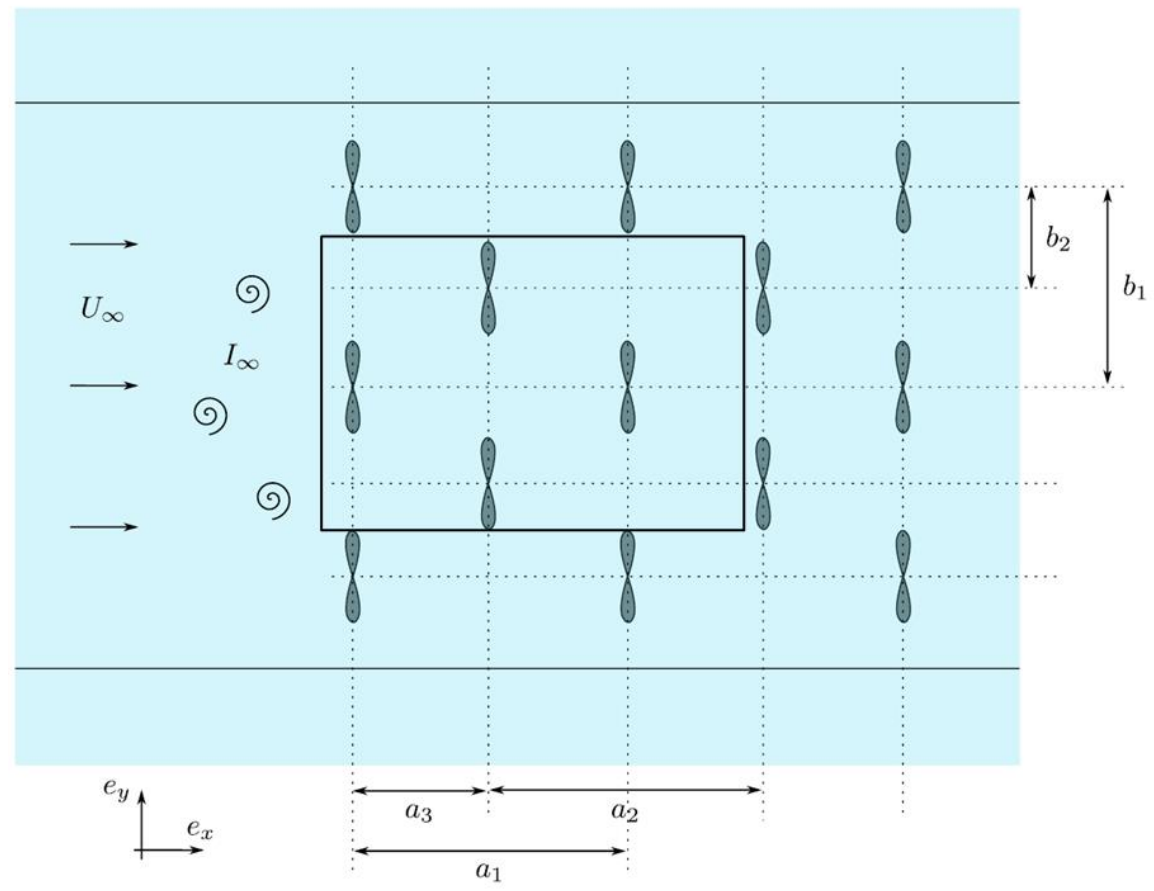

1

(b)

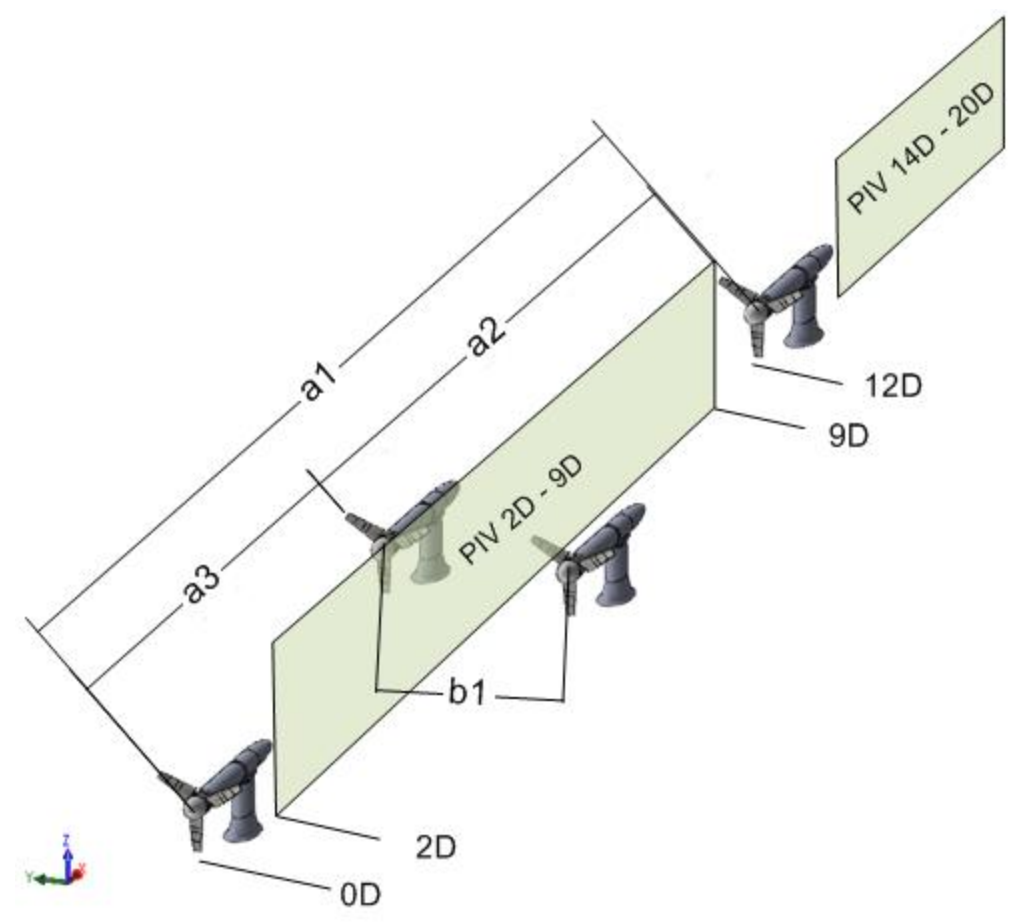

3 Fig. 2 - (a) Typical Array Formation (from [20]) with Experimentally Tested Array Section

4 highlighted (box), (b) Experiment Configuration and Location of Laser Sheet at Array Centre

5 Line for PIV measurements 


\section{$1 \quad 2.1$ Experiment Conditions}

2 Due to the large water depth in relation to the turbine diameter $($ Depth/Diameter $=5.7)$ the set-

3 up does not represent a likely deployment of actual turbines in first/second generation array

4 environment in shallow waters, however takes into account bottom fixed turbines with support

5 structure operating in the lower part of the water column where velocity shear of the ambient

6 flow across the turbine diameter exists. The proximity to the seabed is thus maintained while

7 free surface effects are ignored. The effect of varying device spacings can therefore be

8 investigated with a fixed vertical separation from turbine to sea-bed.

9 Locations within the bottom part of the tidal domain have previously shown to affect the wake

10 shape downstream of tidal turbines by shifting the of wake centreline down due to the velocity

11 shear in ambient flow and reduced wake re-energisation, especially for increased roughness on

12 the seabed, on the lower part of the wake [26].

13 Blockage ratio of a single turbine facing the incoming flow are very low due to the high water

14 depth required to run the experimental facility. The blockage ratio in this experiment for an

15 isolated turbine is $1.3 \%$ and for the full array, with the projected area of three turbines facing

16 the oncoming flow, occupying less than $4 \%$ of the channels cross section, thus no further

17 blockage correction is applied.

18 Table 1 -Tidal turbine characteristics. All values given for CWC Array tests, brackets indicate design range

Model Details Circulating Water Channel

\begin{tabular}{r|l} 
Blade Profile & NREL S814 \\
No. of Blades & 3 \\
Diameter (D) & $0.28 \mathrm{~m}$ \\
Hub height & $0.34 \mathrm{~m}[0.2-0.34]$ \\
Hub diameter & $0.06 \mathrm{~m}$ \\
Hub length & $0.558 \mathrm{~m}$ \\
Pitch Angle & $8.33^{\circ}\left[0^{\circ}-10^{\circ}\right]$ \\
Ratio (TSR) & 4
\end{tabular}



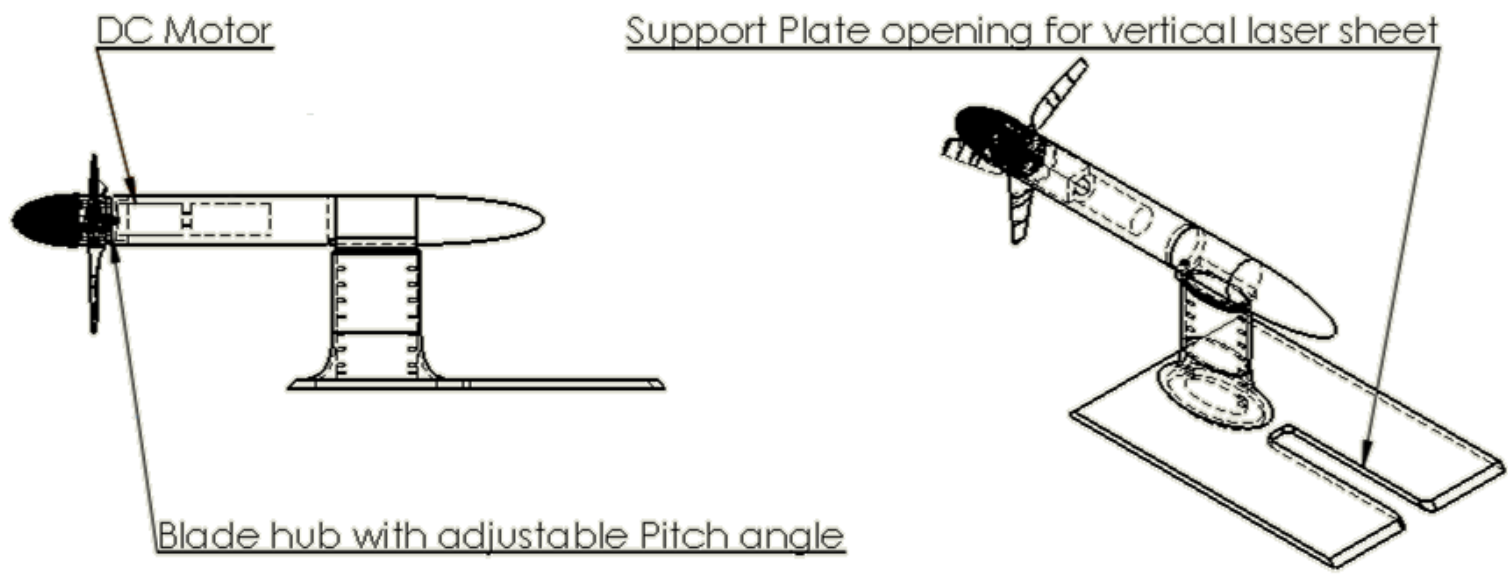

1

\begin{tabular}{lllllllll}
\hline r/R & $\mathbf{0 . 3}$ & $\mathbf{0 . 4}$ & $\mathbf{0 . 5}$ & $\mathbf{0 . 6}$ & $\mathbf{0 . 7}$ & $\mathbf{0 . 8}$ & $\mathbf{0 . 9}$ & $\mathbf{1}$ \\
\hline Chord length (mm) & 42.04 & 39.05 & 36.03 & 33.03 & 29.53 & 27.01 & 24 & 21 \\
Pitch Angle (Degree) & 23.33 & 15.83 & 12.33 & 10.33 & 8.33 & 7.93 & 7.03 & 6.33
\end{tabular}

Fig. 3 - CAD Model of Tidal Turbine Design

\subsection{Model Description}

Four small scale $\left(1 / 70^{\text {th }}\right)$, three bladed tidal turbine models with bottom mounted support have been designed (Fig. 3) and built to conduct experiments in a number of experimental test facilities. The model was designed to allow for change in rotor height and features a pitch variation mechanism to adjust pitch angles \pm 10 degrees, further details of the devices are provided in Table 1.

Array experiments were conducted on a dedicated support frame (Fig. 4) that allows variation in inter-device spacing for both, transverse (b1) and longitudinal (a3) direction while the distance from first to last turbine (a1) remained constant at 12D. Arrays are named according to their longitudinal and transverse spacing denoted by $\mathrm{L}(\mathrm{a} 3)$ and $\mathrm{T}(\mathrm{b} 1)$ respectively, thus the array in Fig. 4 is denoted L5T3.

Table 2 - Geometry of scaled NREL S814 rotor 


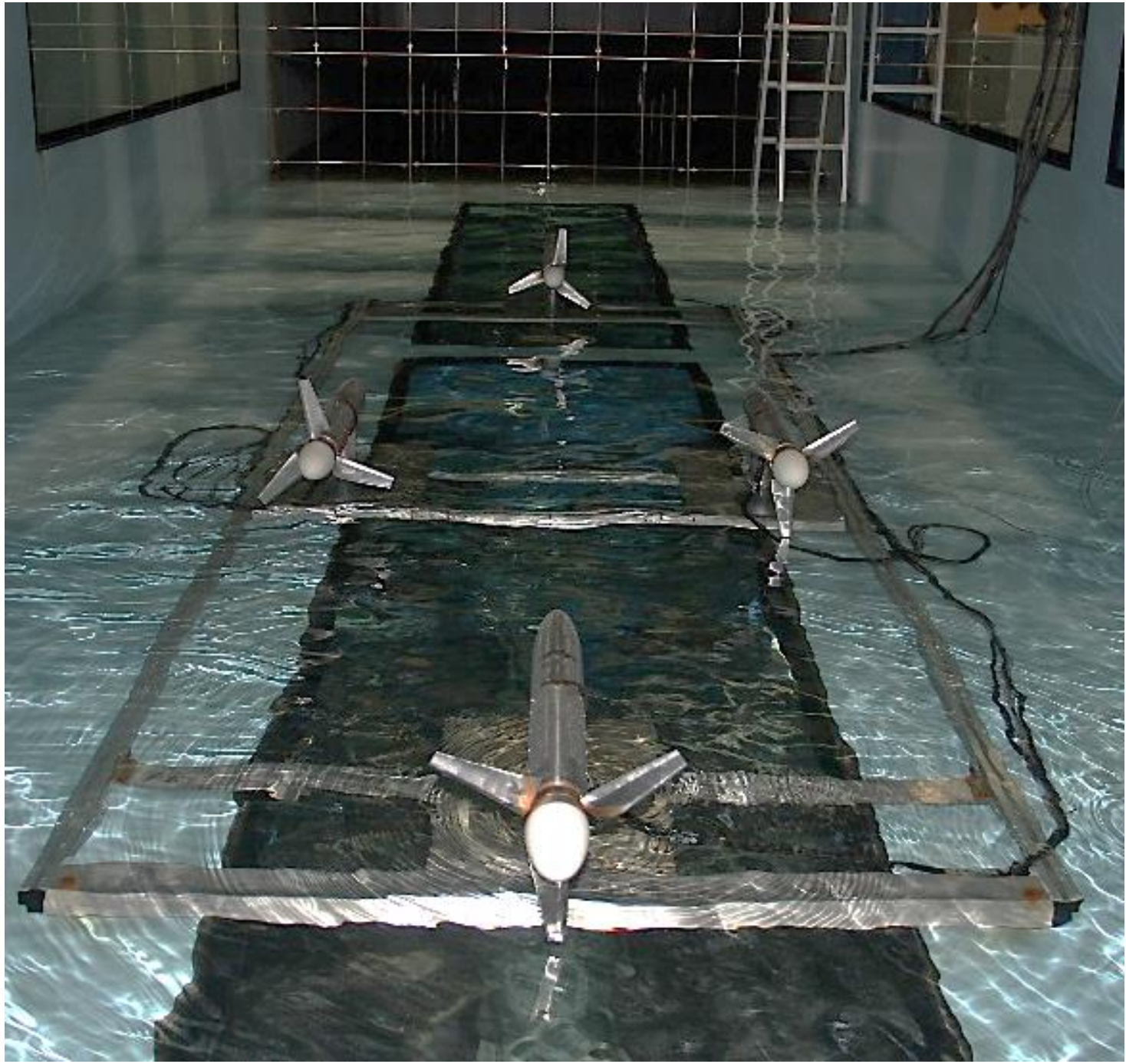

2 Fig. 4 - Array support frame in CWC with Turbine Device Spacing a3 $=5 \mathrm{D}$ and b1 $=3 \mathrm{D}$

3 The blades are based on the NREL S814 section and have been geometrically scaled to a

4 diameter of $0.28 \mathrm{~m}$ with chord and pitch distribution shown in Table 2 . The blade section was

5 chosen because of its robust characteristics in terms of maximum lift coefficient that are less

6 sensitive to surface roughness and operation in unsteady inflow conditions thus providing a

7 good basis for generalised wake characterisation in tidal turbine arrays where changes in

8 surface roughness due to abrasion may play a significant role in reducing the performance of

$9 \quad$ tidal turbine blades. 
1 The blade diameter has been constrained by the use of a long wave-current flume with a water

2 depth of $0.56 \mathrm{~m}$. Kinematic similarity is maintained through the tip speed ratio (TSR), $\lambda=\Omega \mathrm{R}$

3 / $\mathrm{U}_{\infty}$, where $\mathrm{U}_{\infty}$ is defined as the ambient velocity upstream of the tidal turbine array, $\mathrm{R}$ is the

4 radius of the rotor and $\Omega$ the angular velocity $(\mathrm{rad} / \mathrm{s})$. For accurate control and recording of

5 turbine rotation each model is equipped with a Panasonic Minas A5 II motor combined with a

6 control unit that allows individual control of each turbines rotation.

7 The rotation of the turbine rotor is set in the control software and the rotor is then driven to

8 achieve the required TSR. The TSR is kept constant across all tests shown here at TSR $=4$ to

9 test the turbine at optimum design condition and allow for comparison to previously conducted

10 experiments with the same blade section design. Thus the rotational speed was adapted to

11 account for the different ambient current conditions.

12 The performance of the applied rotor design has been previously investigated and presented in

13 [27] for a slightly larger model turbine achieving highest power coefficient (CP) of 14 approximately 0.44 for TSR ranging from approximately $3.2-4$ with the optimised pitch angle of $8.33^{\circ}$ and a thrust coefficient (CT) of approximately 0.95 . The control system was used to ensure constant TSR according to the maximum CP in this experimental set-up. Torque

17 recordings for array test L3T15 have been investigated by comparing the mechanical torque

18 required to maintain the desired rate of rotation without any current present, to the torque

19 applied to maintain the TSR when a current is acting on the turbine device. The torque recordings in Fig. 5 show that for the first turbine in array, on average approximately $44 \%$ of

21 the required torque was generated by the current therefore, considering differences in

22 evaluating power matching the $\mathrm{CP}$ of previous experiments, whereas the downstream turbine

23 averaged approximately $30 \%$ and showing much larger fluctuations due to operating in the

24 wake of the upstream turbines. 


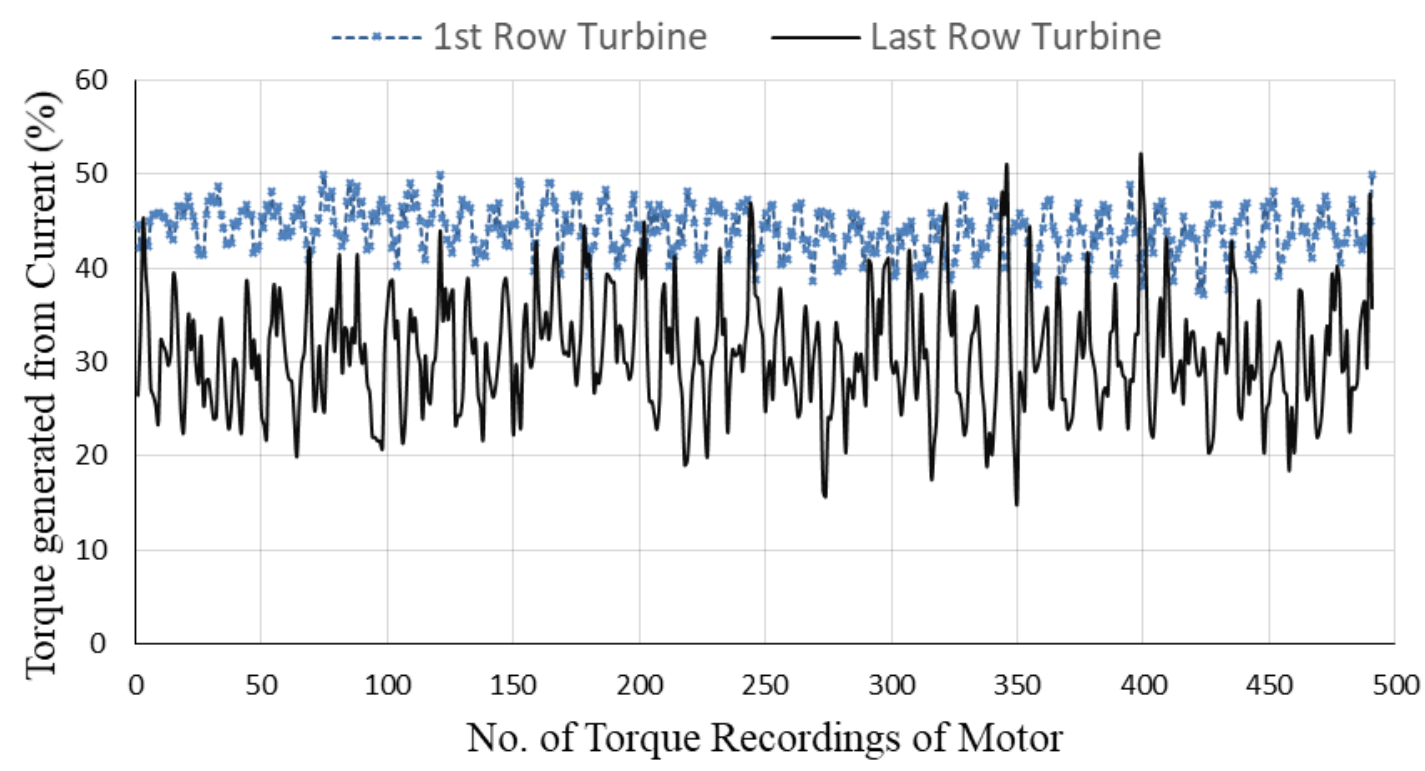

Fig. 5 - Comparison of torque generated by current as percentage of torque required to maintain rotation according to desired TSR (4) without any current acting on the tidal turbine structure

The choice of characteristic length for calculation of Reynolds number ( $\mathrm{Re})$ varies across previous tidal turbine experiments. Reynolds numbers have been calculated using the free stream current velocity as well as tip speed of the rotor and used diameter [19], radius [17, 18] and chord length [28] at different stations as characteristic length. Maintaining dynamic similarity between small scale experiments and full scale tidal turbines is difficult with geometrically scaled blade sections, though in this study Reynolds numbers have exceeded the laminar-turbulent threshold of approximately $2 \times 10^{3}$ for all reference lengths as shown in

Table 3. The Reynolds independence threshold for main flow statistics was reported from experimental wind turbine tests to be $4.8 \times 10^{4}$ for mean velocity and $9.3 \times 10^{4}$ for higher order statistics [29] which has been exceeded based on the turbine diameter and current inflow in all but one test case presented herein. It was also highlighted that the sensitivity to Reynolds number decreases with increasing distance from the turbine. From numerical investigation of non-dimensional scaling of tidal turbine devices Reynolds independence was $5 \times 10^{5}$ [30] for the specific tidal turbine geometry and pitch angle. 
1 Based on the scaling for small scale tests, the Reynolds number is maximised to achieve the

2 required turbulent flow classification while maintaining similarity of the non-dimensional

3 kinematic scaling parameters, i.e. Froude scaling the approach flow and matching TSR at

4 rotational speeds within the range of previous experiments of small tidal turbine rotors while

5 avoiding excessive rotor rotation $[19,26,31]$.

6 All 4 models are operating at the same centre-line height of $0.34 \mathrm{~cm}$ from the test section

7 bottom. This results in a rotor-tip to seabed separation of $0.71 \mathrm{D}$, while turbine tip-free surface

8 separation is 4D. For the purpose of wake measurements and characterisation of the local flow

9 within the array the origin of the coordinate system is placed at the position of the first rotor,

10 with the in stream direction denoted by $\mathrm{x}$, the cross-stream direction as $\mathrm{y}$ and the vertical

11 position by $\mathrm{z}$. The corresponding velocity components are denoted as Ux and Uz for the

12 instream and vertical flow components respectively.

\subsection{PIV Measurements}

14 The LaVision GmbH PIV system used to record images of illuminated seeding particles in the

15 wake of the tidal turbine array included a double pulsed Nd: YAG laser, located on a traversing 16 unit below the centre line of the test section for accurate positioning, with output energy of $17425 \mathrm{~mJ} /$ pulse at $532 \mathrm{~nm}$ wavelength to illumitate the particles. Two LaVision ImagerProX11M 18 CCD cameras used in a stereoscopic arrangement to record double frame/ double exposure 19 images of the illuminated field of view at the maximum frequency of $4.52 \mathrm{~Hz}$ (Fig. 8). 500 20 images were collected at each location (Fig. 9) throughout the array, controlled and processed 21 in DaVis 8.2.2 to yield vector maps of the flow for further analysis. To ensure 500 images are 22 sufficient for the time averaging of flow characteristics the moving average of the streamwise and vertical velocity components and RMS values has been monitored and are presented in

24 Fig. 10. Analysis has been performed for stereo and mono PIV applying image correction based on the PIV system's calibration conducted beforehand. 
1 Images were pre-processed using a sliding background filter to remove large intensity

2 fluctuations due to reflections in the background of the image and particle intensity

3 normalisation to achieve more homogeneous particle intensitities, allowing smaller particles to

4 contribute to the cross-correlation.

5

Table 3 - Reynolds numbers with different characteristic length definitions

\begin{tabular}{lcc} 
Characteristic Length & Array $-\mathbf{0 . 4 4} \mathbf{~ m} / \mathbf{s}$ \\
\hline Diameter & $\operatorname{Re}\left(\mathrm{U}_{\infty}\right)$ & $\operatorname{Re}\left(\mathrm{U}_{\text {tip }}\right)$ \\
Radius & $1.23 \mathrm{E}+05$ & $4.93 \mathrm{E}+05$ \\
Chord (0.5R) & $6.16 \mathrm{E}+04$ & $2.46 \mathrm{E}+05$ \\
Chord (0.7R) & $1.58 \mathrm{E}+04$ & $6.34 \mathrm{E}+04$ \\
Chord (tip) & $1.30 \mathrm{E}+04$ & $5.19 \mathrm{E}+04$ \\
\hline
\end{tabular}

7

8 To improve vector calculation in areas with large velocity fluctuations vertically across the

9 wake of tidal turbines, calculations are performed using multiple iterations with decreasing 10 interrogation window size $(64 \times 64$ to $32 \times 32 \mathrm{px})$ and adaptive gaussian weighting function, 11 automatically adjusting window size and shape to local seeding density and flow gradients.

12 Data extraction for comparison is done along the wake centre line at hub height as indicated in

13 Fig. 6 a) below, showing the array centre line passing between the second row turbines for the 14 array configuration L3T15, the missing data in the corners of the recording window due to the 15 camera lens and intensity of the laser light can be clearly seen here and in the resulting 16 combined wake plots in Fig. 6 b). The configuration for all L5 arrays is shown in Fig. 7, where 17 an obstruction to the laser sheet was created by the position of the laser under the test section 18 of the CWC, those points have been excluded from the results discussion. 
(a)

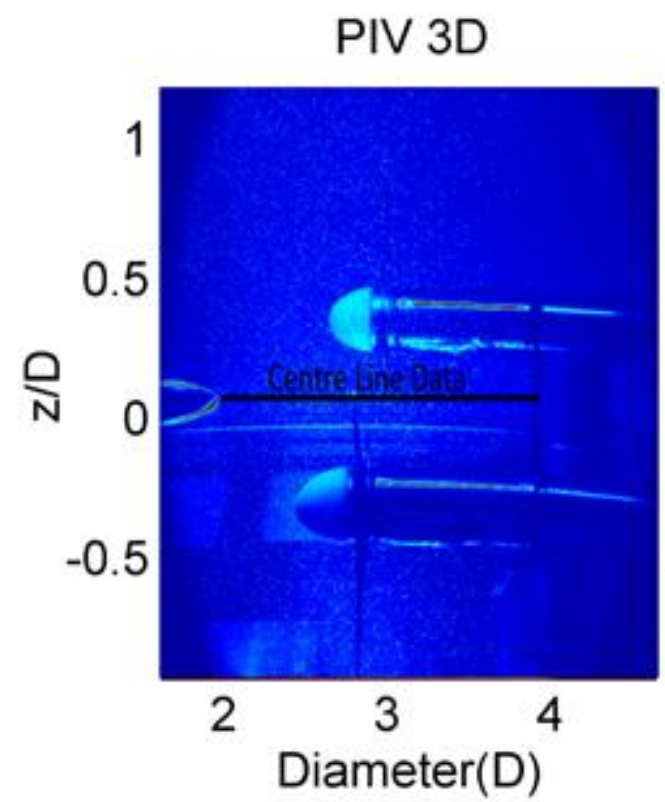

(b)

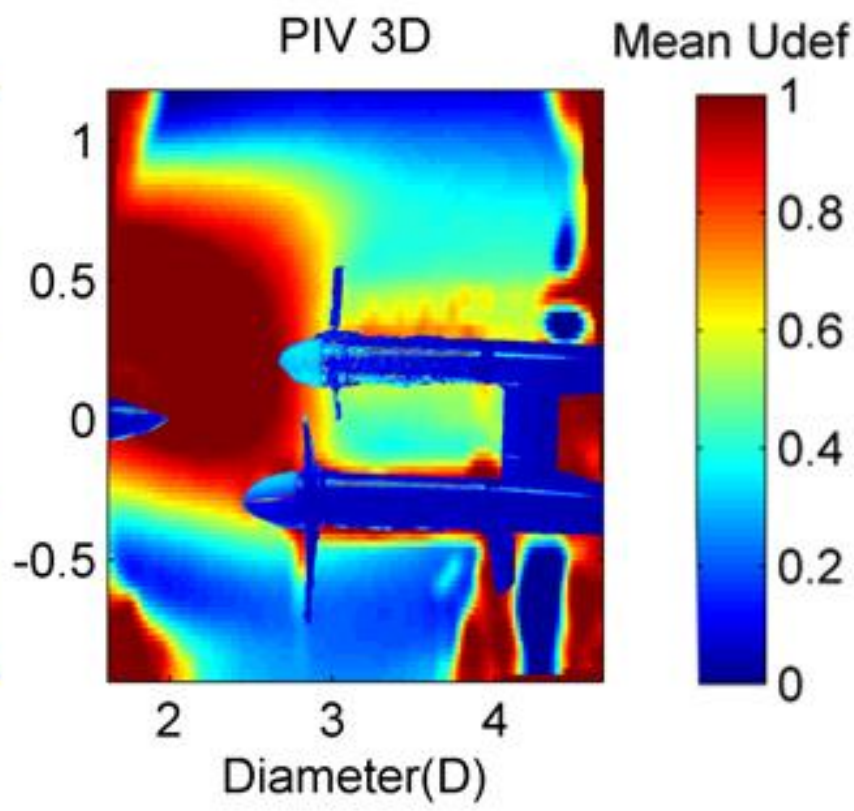

Fig. 6 - (a) Raw Image, and (b) Contour Plot of Velocity Deficit Showing the Influence of the

Turbine Devices Located Offset from the Array Centreline in Row Two for L3T15.

(a)

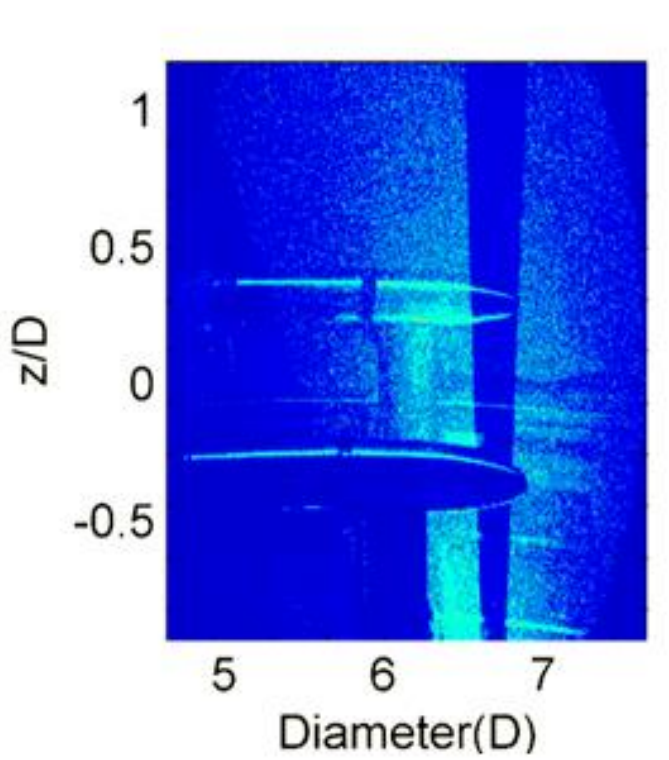

(b)

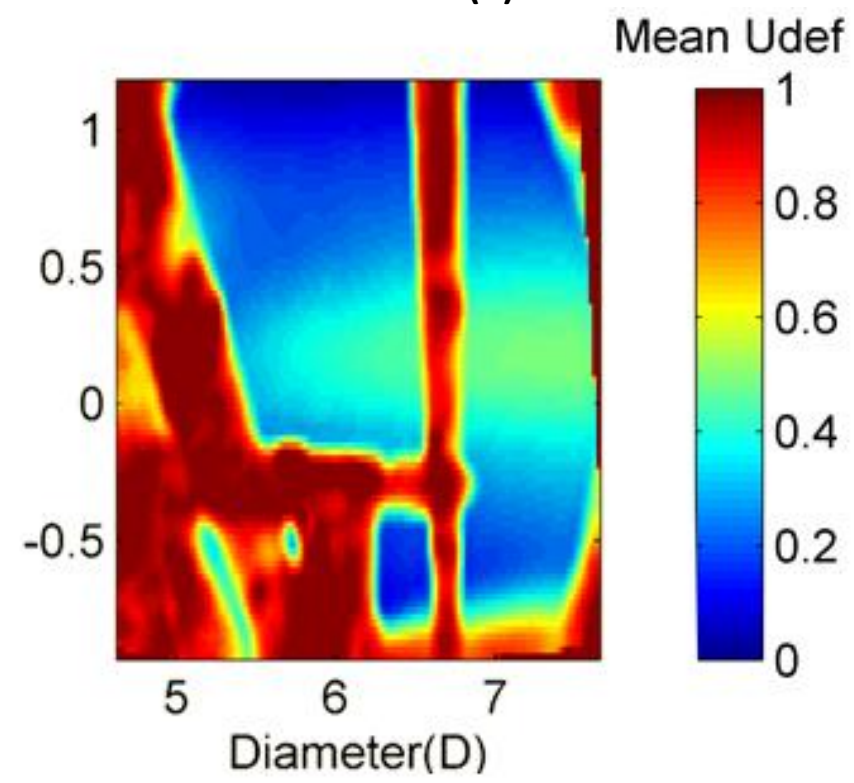

Fig. 7 - Location of Row Two Turbines in L5 Array Tests Shown are (a) Raw Image and (b) Resulting Contour Plot of Velocity Deficit Shown to Highlight the Interference with the Support Structure. 


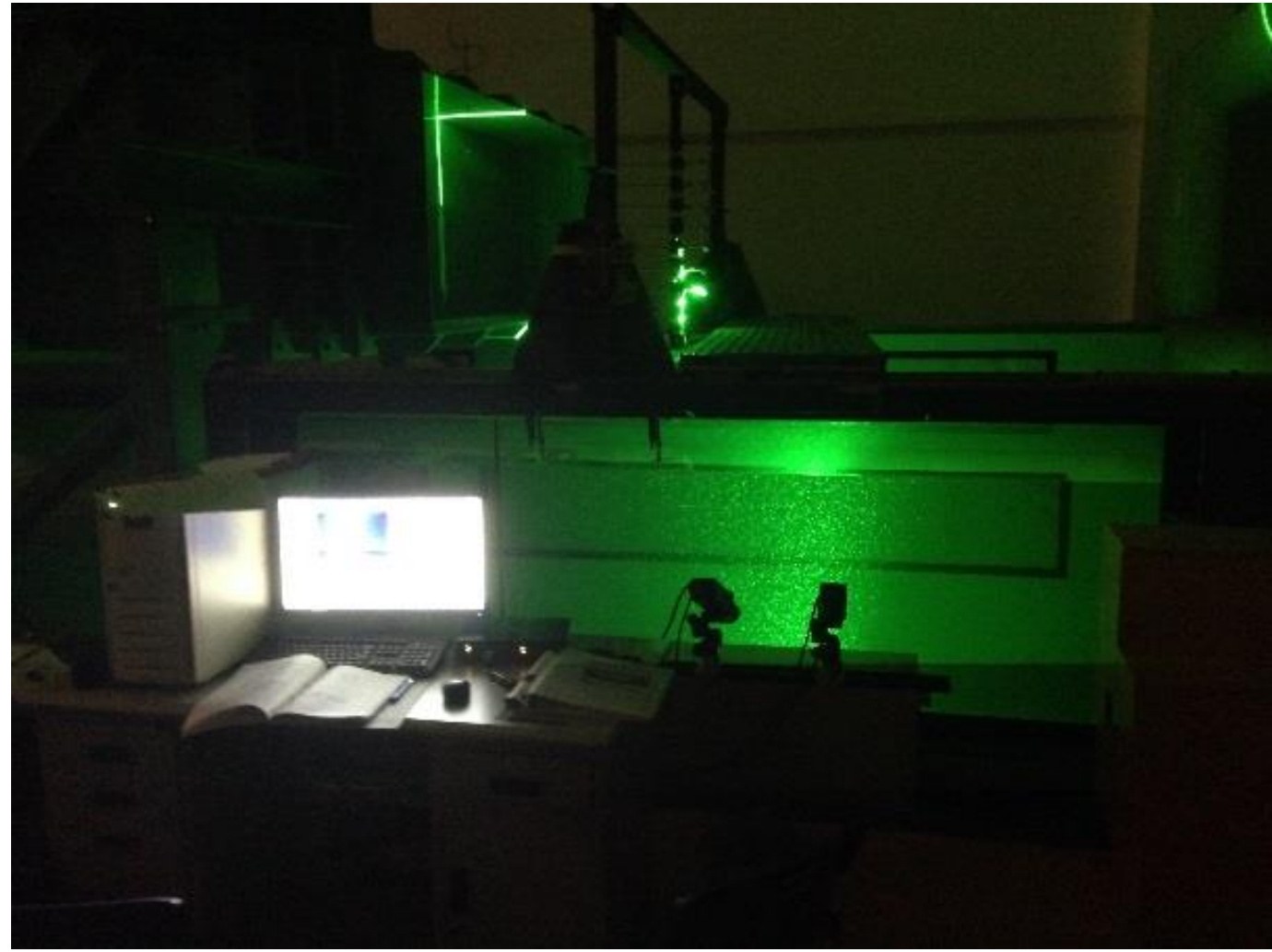

Fig. 8 - Experiment PIV set-up at Shanghai Jiao Tong Circulating Water Channel

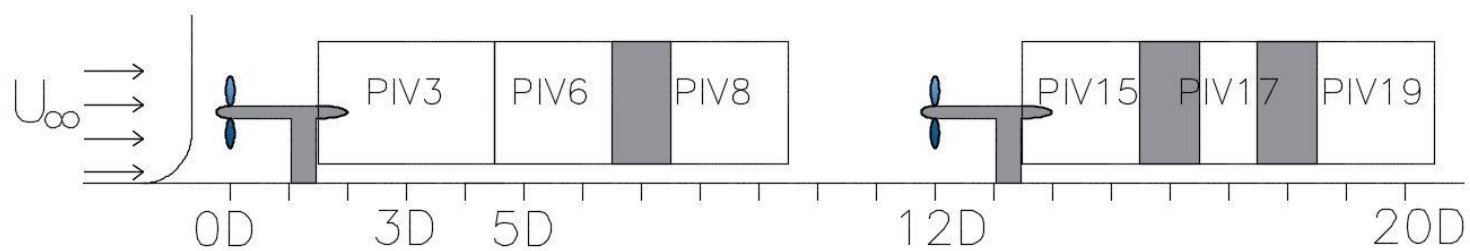

3
4

Fig. 9 - Measurement locations at array centre line (overlapping areas shaded)

5

(a)

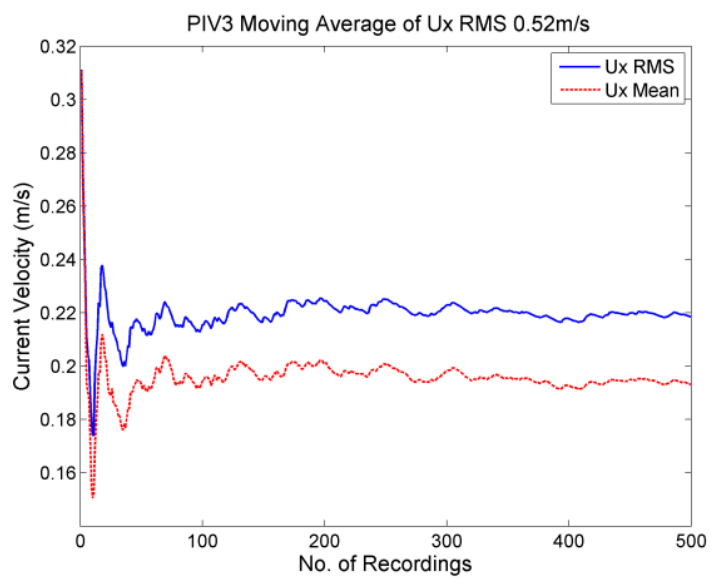

(b)

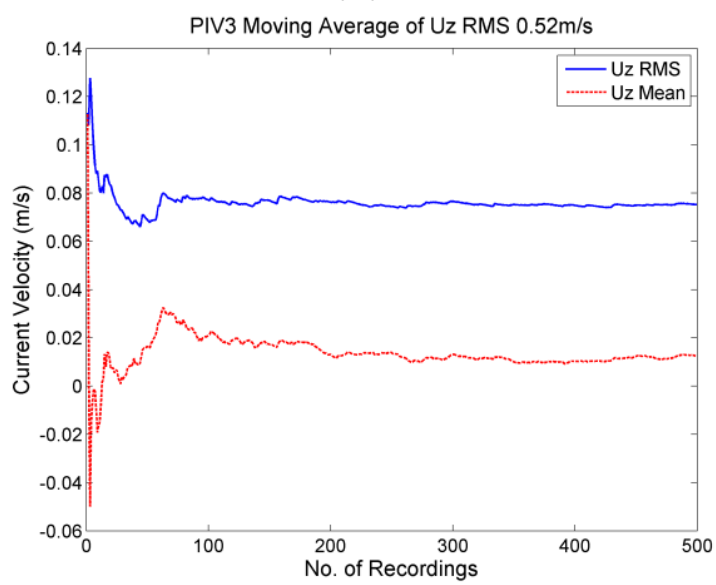

Fig. 10 - Moving average of a) Ux and b) Uz and RMS value at PIV3 for L3T15 array 


\section{$1 \quad 3$. Results \& Discussion}

2 Results are presented using measured velocity and calculated two-dimensional turbulence

3 intensity (1) along the wake centre-line and the axial wake velocity deficit (2) is used for single

4 turbine and 3 turbine experiment. The velocity deficit is also used for all 4 turbine arrays to

5 compare the reduction and recovery of the flow velocity within the wake with reference to the

6 entire array section and the undisturbed upstream flow. The inflow conditions upstream of the

7 array are used for determining operating conditions of multiple in-line arranged turbines as

8 applied in [20], hence describing the overall array behaviour and thus showing increased

9 velocity deficits within the wake of the downstream turbine. Trends in the velocity deficit

10 recovery however will reflect the local flow re-energisation within the wake of downstream

11 turbines. The velocity deficit shown downstream of the array thus represents the resulting array

12 wake of the tested section.

13 This notation does also affect the actual TSR the downstream turbine is run at, which will be

14 increased due to being located in the wake of the upstream turbine, the selected airfoil sections

15 as described earlier and pitch degree of $8.33^{\circ}$ have been shown to have small variations in the

16 turbine performance across a range of operating conditions with a CP exceeding 0.4 for TSR

17 ranging from 2.8 to 4.75 and 0.3 up to a TSR of 6 , with CT remaining practically constant

18 between TSR $4-7$ which lie within the expected TSR for the downstream turbine when

19 operating in the wake of the upstream turbine [27]. 


\subsection{Single Device Wake measurements}

2 Prior to assessing the wake within an array of four tidal turbines, a single turbine has been

3 tested in a range of currents with the same TSR. The centreline axial wake velocity deficit for

4 a single turbine model operating at three different current settings in the CWC is shown in Fig. 11.

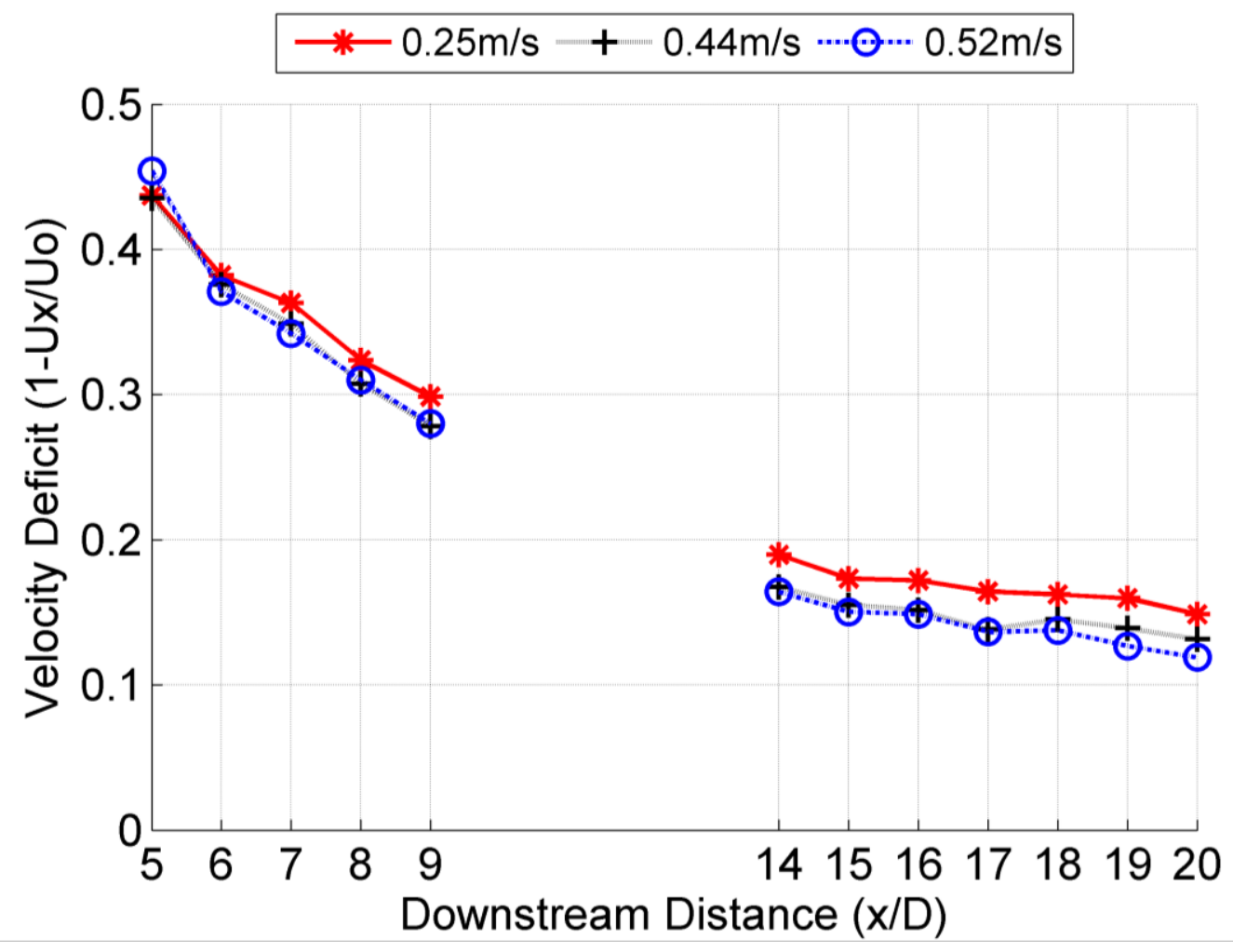

Fig. 11 - Centreline wake velocity deficit for single turbine

8 The measured wake velocity deficit Fig. 11 recovers at a higher rate in the near wake of the

9 turbine, where turbulence levels are high (see Fig. 12) due to the combined presence of rotor

10 and support structure. With increasing distance downstream of the rotor, the velocity recovery

11 at the centre line slows down due to dissipation of turbulent kinetic energy. In the far wake of the single turbine configuration, the wake centre-line velocity recovers slower and reaches approximately $85 \%$ of the free stream velocity at $20 \mathrm{D}$ downstream of the rotor. The evolution of velocity deficit through the wake, from $44 \%$ at $5 \mathrm{D}$ to $28 \%$ at $9 \mathrm{D}$ is comparable to previous single turbine experiments conducted at similar ambient conditions. Mycek, Gaurier, Germain,

16 Pinon and Rivoalen [17] showed a reduction in centre-line velocity deficit from 50\% at 5D to 
$125 \%$ at $10 \mathrm{D}$ for a slightly higher ambient turbulence intensity flow (3\%). Other experiments at

2 slightly higher ambient turbulence intensities $[22,32]$ of $10 \%$ and $8 \%$ respectively have shown

3 recovery of the deficit by about $20 \%$ between $5 \mathrm{D}$ and $10 \mathrm{D}$.

4 The calculated centre-line turbulence levels (Fig. 12) in the near wake up to 10D decrease from $522 \%$ to $12.5 \%$, almost identical to those measured in [17]. With increasing distance from the 6 rotor (14D-20D), recovery towards ambient conditions slows down, and turbulence intensity

7 remains at considerably higher levels of approximately 3-4 times the ambient upstream 8 conditions of $2 \%$.

9 The velocity deficit (Fig. 11) shows small variation across the different current velocities of a 10 single turbine operating at constant tip-speed ratio for currents of $0.44 \mathrm{~m} / \mathrm{s}$ to $0.52 \mathrm{~m} / \mathrm{s}$. With a 11 current velocity of $0.25 \mathrm{~m} / \mathrm{s}$ the resulting Reynolds number is below the independence threshold 12 for the main wake statistics as presented in [29], therefore the deviation of the resulting wake 13 velocity deficit could be attributed to this. A further consideration at this small scale is the very 14 high rotational velocity required to maintain dynamic similarity between the scaled model and proposed full scale tidal turbines that is affecting the momentum extraction and inducing rotational flow effects that influence the development of the wake.

(a)

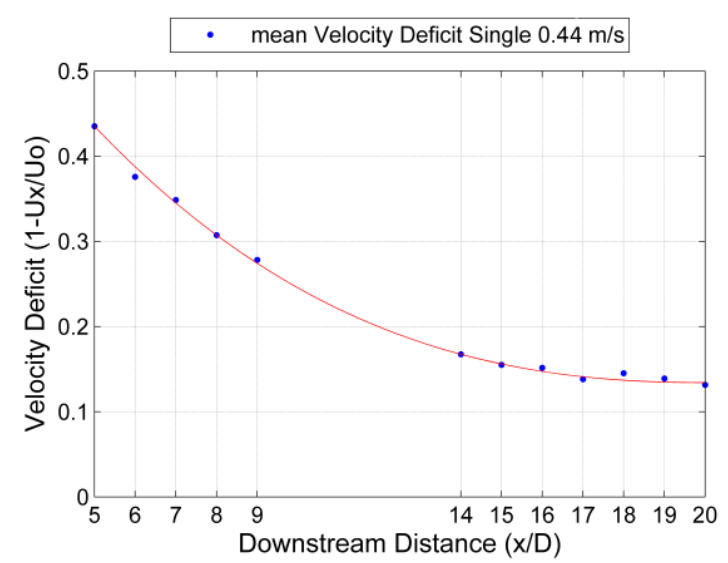

(b)

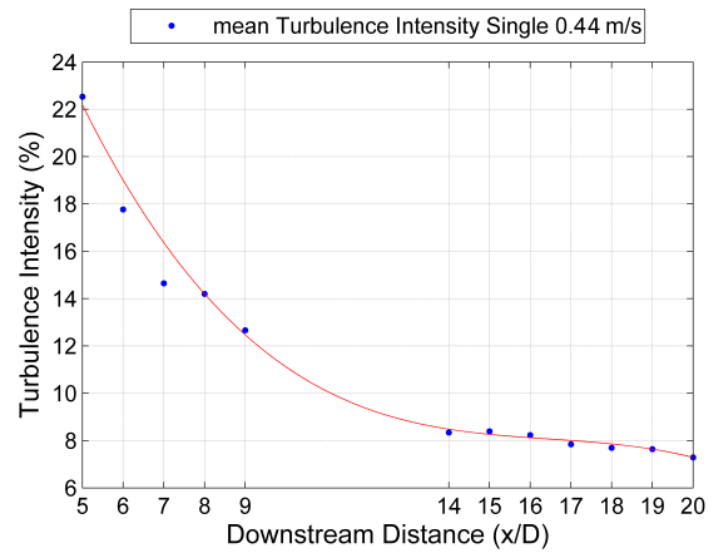

Fig. 12 - a) Velocity Deficit and b) Turbulence Intensity Downstream of Single Turbine 


\section{$1 \quad 3.2$ Centre - line wake in 3 Turbine staggered arrangement}

2 The effect of adding an additional row of turbines with centre-line offset of $\pm 3 \mathrm{D}$ on the centre-

3 line wake is investigated. The measured time-averaged velocity deficit (Fig. 13) at 5D is lower

4 for the 3 Turbine arrangement (30\%) than the single turbine velocity deficit of $40 \%$, however

5 trends in recovery are very similar to the single turbine arrangement in the region from 5-10D.

6 By adding a second row of turbines, the near wake characteristics (between 5D and 9D) show

7 a lower velocity deficit compared to the single turbine case (29\% compared to $43 \%$ ).

8 An increase in velocity deficit is observed at $0.44 \mathrm{~m} / \mathrm{s}$ between $15 \mathrm{D}$ and $19 \mathrm{D}$ along with

9 turbulence intensity that increases slightly between $16 \mathrm{D}$ and $18 \mathrm{D}$. At $0.52 \mathrm{~m} / \mathrm{s}$ ambient current 10 velocity, this effect is less pronounced, however the velocity deficit remains virtually constant

11 between 15D and 18D and continues recovering further downstream where a slight increase in 12 turbulence intensity at $18 \mathrm{D}$ can be observed at $0.52 \mathrm{~m} / \mathrm{s}$ which shows the increased rate of 13 velocity recovery seen in Fig. 13.

14 The wake further downstream is therefore experiencing a reduction in wake recovery, which may indicate the positions where the outer turbine wakes reach the array centre-line, hence slowing down mixing with the ambient flow as these wakes have significantly lower velocities.

17 This evolution of the wake downstream would most significantly affect further positioning of 18 additional tidal turbines in large-scale arrays.

19 The contour plot in Fig. 15 showing the velocity deficit evolution at the centreline of the test section shows increased velocity deficits downstream of the rotor and a reduced velocity deficit in the centre of the wake. The top part of the wake ( $\mathrm{y} / \mathrm{D}>0)$ shows increased recovery of the wake velocity compared to the bottom part of the wake and a small deflection of the wake towards the bottom of the test section is observed, caused by the small shear present in the onset flow (Fig. 1). 
(a)

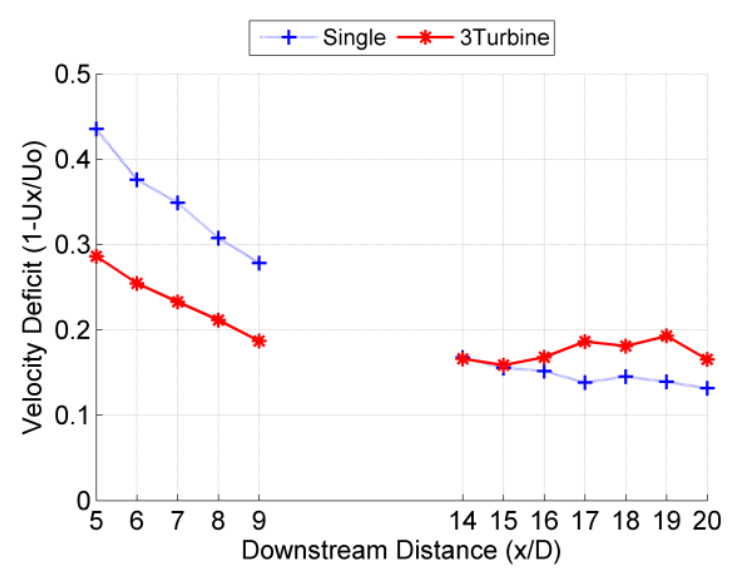

(b)

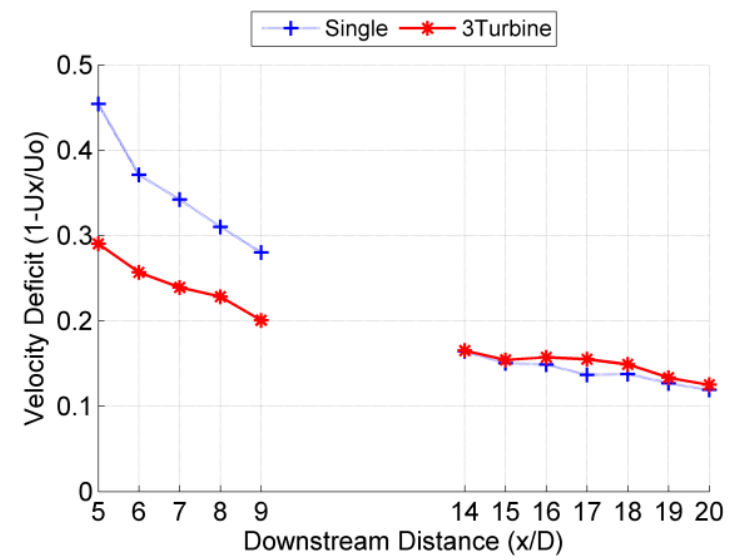

Fig. 13 - Velocity Deficit Comparison Between Single Turbine and 3 Turbine Arrangement at a) $0.44 \mathrm{~m} / \mathrm{s}$ and b) $0.52 \mathrm{~m} / \mathrm{s}$ Ambient Current Velocity

(a)

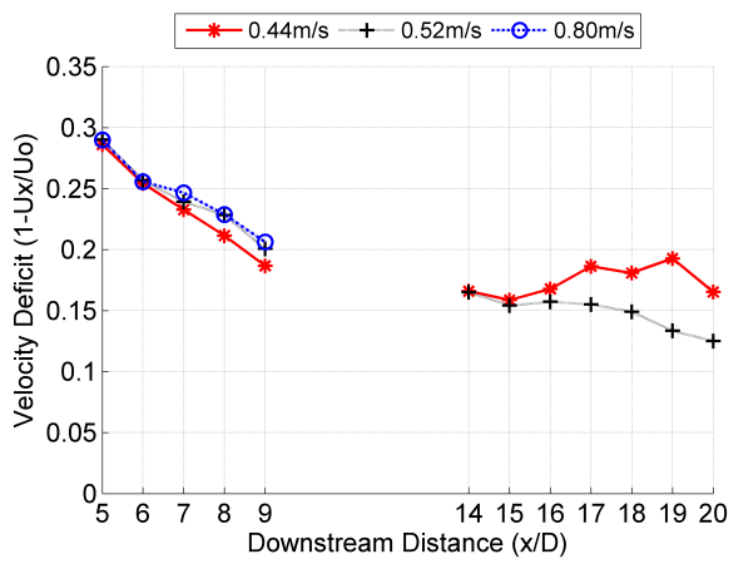

(b)

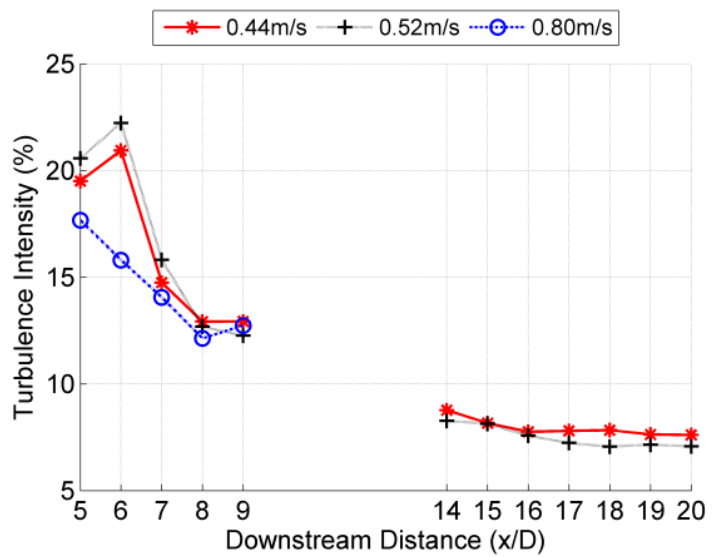

Fig. 14 a) Velocity deficit and b) Turbulence Intensity Comparison of 3 Turbine Arrangement at Different Current Velocities

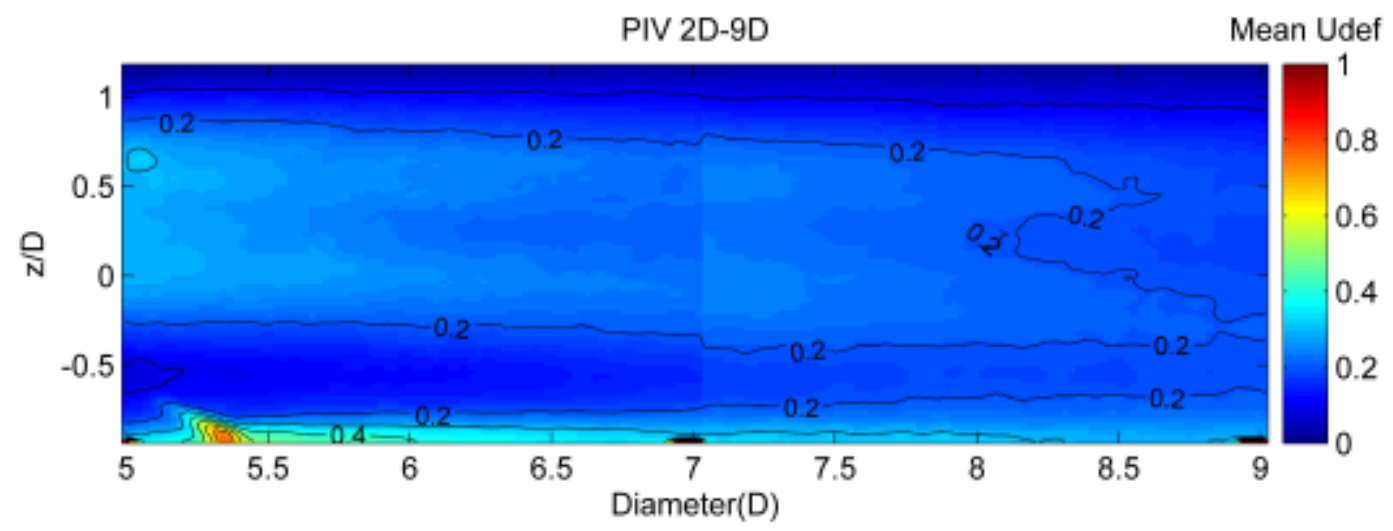

Fig. 15 - 3 Turbines Velocity Deficit (0.44m/s Current) Downstream of 2nd Row 
$1 \quad 3.3$ Tidal turbine array tests

2 To determine the effects of multiple turbines operating in close proximity, measurements have

3 been taken at the centre-line of the proposed array sections. Within the array, measurements

4 downstream of turbine A to upstream of turbine D (representing downstream distances of 2D

$5-9 D)$ and measurements downstream of turbine D (14D - 20D) are used to investigate velocity

6 and turbulence characteristics across a number of array configurations. Data points between

$7 \quad 2 \mathrm{D}$ and 5D for close lateral spacing are affected by the presence of support structure in the PIV

8 recordings, which can be observed in the contour plots (Fig. 21 \& Fig. 22).

9 Fig. 16 shows a comparison of time averaged velocity deficits for both longitudinal spacings

10 tested. There is a clear correlation between the lateral spacing of turbine B and D and the

11 evolution of the velocity deficit as smaller spacing leads to higher velocity deficits remaining

12 in the wake, as well as slower recovery towards free- stream velocity especially between 5D -

13 9D. The velocity deficit levels observed for array tests have been considerably higher,

14 especially for close lateral spacing of $1.5 \mathrm{D}$ between the middle row turbines. For both

15 longitudinal distances velocity recovery slows down approximately 2D - 3D downstream of

16 the second row turbines. An increase of centre-line velocity deficit is observed between 5D and

17 9D for the close transverse and increased longitudinal spacing of L5T15 array. These indicate

18 that the close transverse spacing reduces wake recovery at the centre line and could be

19 explained with the combination of adjacent wakes to form a wide, single wake reducing the

20 mixing with ambient flow at the centre line for wake re-energization.

21 The wake downstream of the array shows smaller variations between the different configurations, however with larger spacing between the upstream turbines B \& D, the rate of velocity recovery of the array wake is increased and velocity deficit remain smaller for a lateral

24 spacing of 3D. Slightly increased velocity deficits remain for lateral spacings of less than 3D

25 in the L5 arrays with a deficit of $35 \%$ at L3 and $39 \%$ at L5 respectively (Fig. 16b). For a 
1 longitudinal spacing of 3D, the velocity deficit is almost identical between 1.5D and 2D

2 transverse spacing. The highest velocity deficit at L5 arrays is seen for lateral spacing of 1.5D.

3 Comparing the array wake velocity recovery downstream of turbine D to the 3 Turbine

4 arrangement shown above, the influence of the middle row turbine on the wake recovery is less

5 pronounced. This could be attributed to the overall high velocity deficit, changes in turbine

6 inflow conditions and the outer turbine wakes preventing ambient flow from reaching the array

7 centre-line as rapidly as in the case without turbine D. Increased velocity recovery for large

8 spacing, inside the array section and downstream of the array would indicate increased mixing

9 with ambient flow and could point towards ambient flow penetrating between the individual

10 wakes. This will be further investigated in three dimensional numerical simulations.

(a)

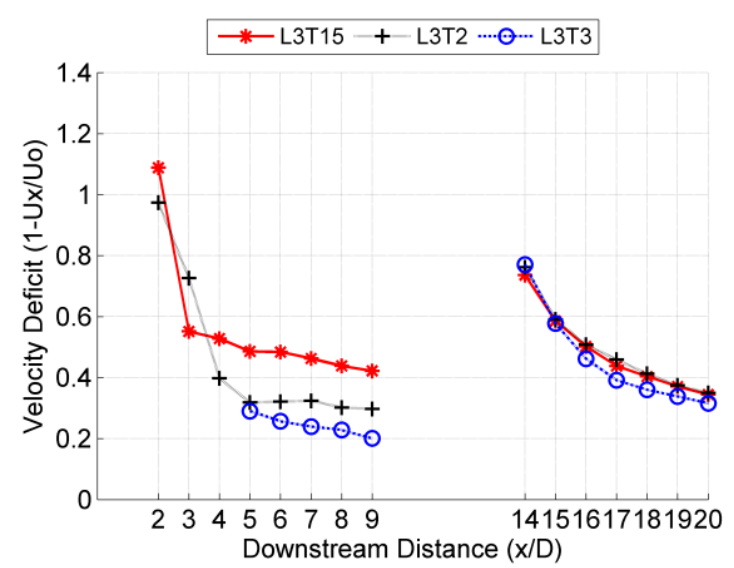

(b)

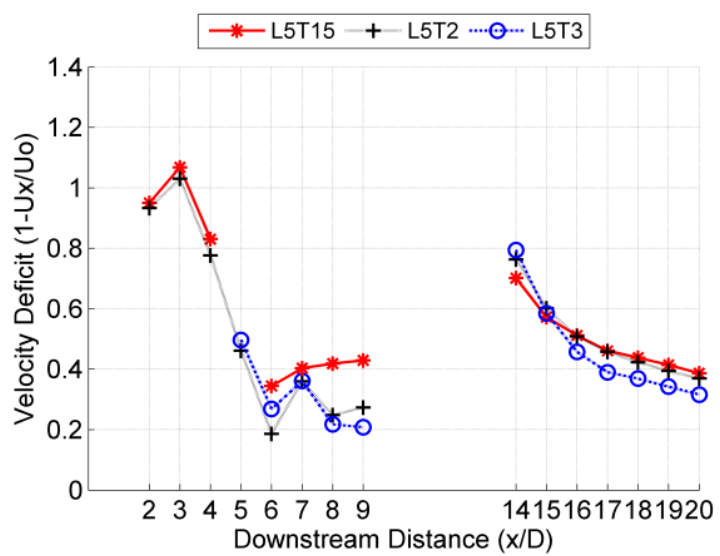

Fig. 16 - Centre-line array wake velocity deficit comparison at $0.52 \mathrm{~m} / \mathrm{s}$ for a) $\mathrm{L} 3$ arrays and b) L5 arrays 
(a)

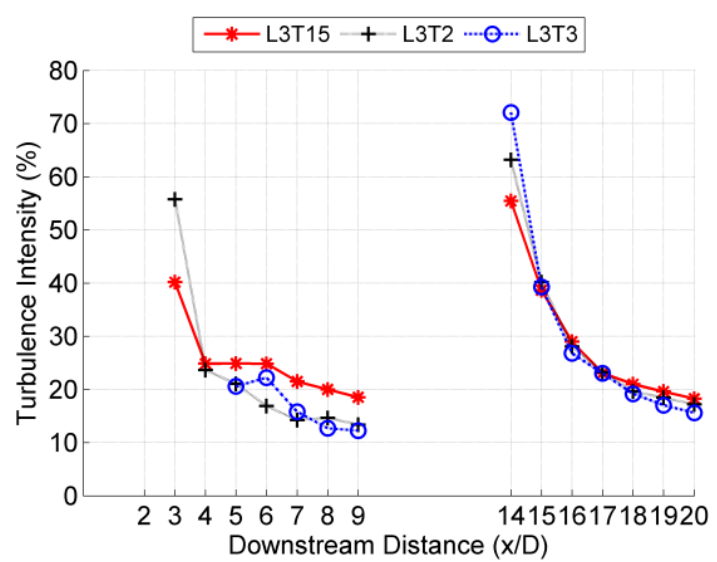

(b)

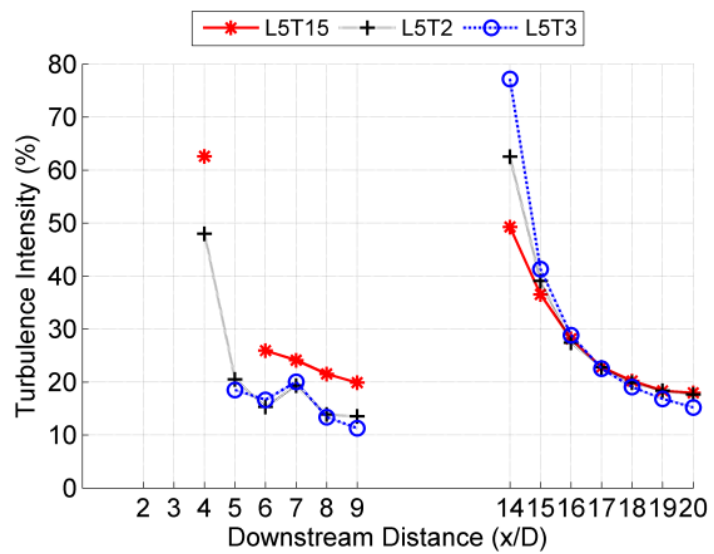

Fig. 17 - Centre-line array wake turbulence Intensity comparison at $0.52 \mathrm{~m} / \mathrm{s}$ a) for L3 arrays and b) L5 arrays

1 From the flow characteristics presented in Fig. 16 \& Fig. 17, it can be seen that increasing

2 lateral spacing leads to significantly improved recovery within the array section tested, hence

3 increasing inflow velocity to the first in-line turbine in such arrangements. For 3D longitudinal

4 spacing, differences in velocity deficit of 13\% between L3T15 and L3T2 (15\% for L5) compared to $9 \%$ between $\mathrm{T} 2$ and T3 (7\% for L5) show a reducing influence of lateral spacing.

6 The downstream array section wake is less influenced by the lateral spacing tested and shows

7 small variations only in terms of wake recovery far downstream, however with the trend of

8 slightly increasing differences between the different formations with increasing lateral spacing.

9 Comparison of the wake velocity deficits downstream of the arrays show improved recovery

10 for increased lateral spacing of the middle row turbines. Some benefits of very close spacing

11 in both longitudinal and lateral direction can be seen in the wake recovery for L3T15 array (Fig.

12 18) showing improved wake recovery at $20 \mathrm{D}$ downstream. The influence of longitudinal

13 spacing reduces with wider lateral spacing of the middle-row turbines when comparing the

14 velocity deficit downstream of the array for the both T3 cases.

15 The downstream turbine operated at increased TSR due to the reduced inflow velocity and showed lower performance with higher variations(Fig. 5) however, the turbine blade design 
1 was shown previously to maintain high and practically constant CT values over a range of TSR

2 expected to be encountered by the upstream and downstream turbine [27]. The detailed

3 performance of array turbines will be further investigated further numerically.

4 The wake recovery for larger transverse spacing could hence be related to more of the ambient

5 flow travelling through the array at higher velocities and being pushed towards the array

6 centreline without entrapping a large volume of slow moving fluid as for the close transverse

7 configurations which effectively creates a single wake with slowed velocity recovery at the

8 centreline as shown for close spacing in [21]. This can be a particular area of interest for the

9 initial rows in large scale arrays where device generated turbulence levels are lower, hence

10 wake recovery would be influenced more by the characteristics of the ambient flow.

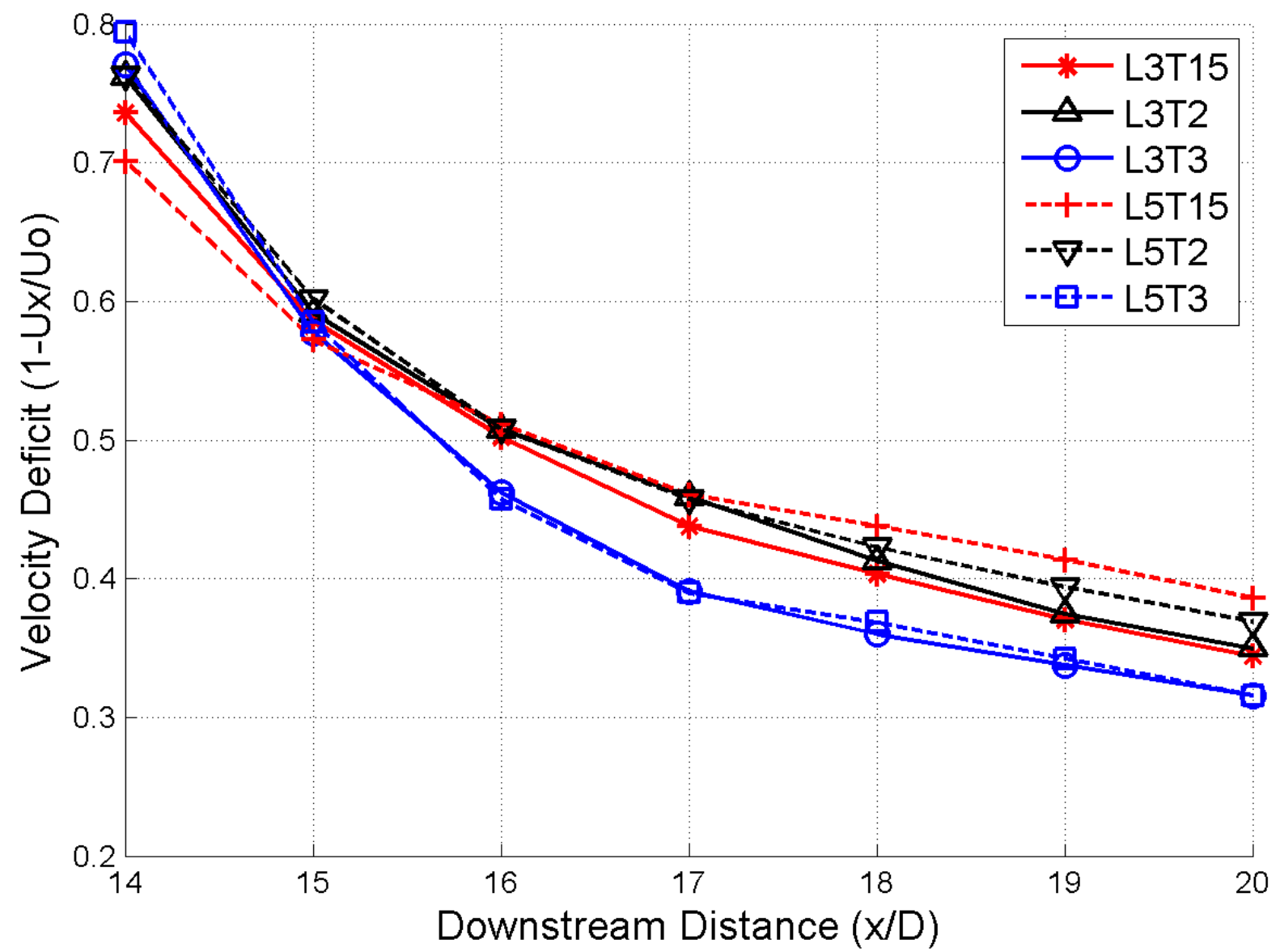

12

Fig. 18 - Comparison of wake velocity deficit downstream of various array configurations wit ambient flow of $0.52 \mathrm{~m} / \mathrm{s}$ 
$1 \quad$ 3.2.1 PIV Flow Visualisation in Array

2 Flow field measurements with PIV provide further insights into the resulting wake without the

3 need for extensive point-by-point measurements along a pre-defined grid of measurement

4 locations. This provides visualized wake data for inspection and determination of the processes

5 occurring at the centre line of different array formations. For visualization, the individual

6 measurements taken have been combined to provide continuous wake field for the inner array

7 field along the centre line (Distances of 2D-9D) as well as the array wake downstream (14D-

8 20D).

9 Vectors are combined by averaging where both images have flow vectors within the overlapped

10 area. Areas where no data has been recorded due to the PIV set-up (no access for laser sheet or

11 reflections of device surface (refer to Section 2.3 ) or where vectors were removed during post-

12 processing remain visible in the resulting contour plots and show small inconsistencies of the

13 flow field around this area as a product of the overlapping. Fig. 19 shows individual

14 measurements for each wake location in a) and the combined wake plot for the wake field

15 between 2D and 9D. The array centre line from which data is presented for the velocity deficit

16 and turbulence intensity is shown. 
a)
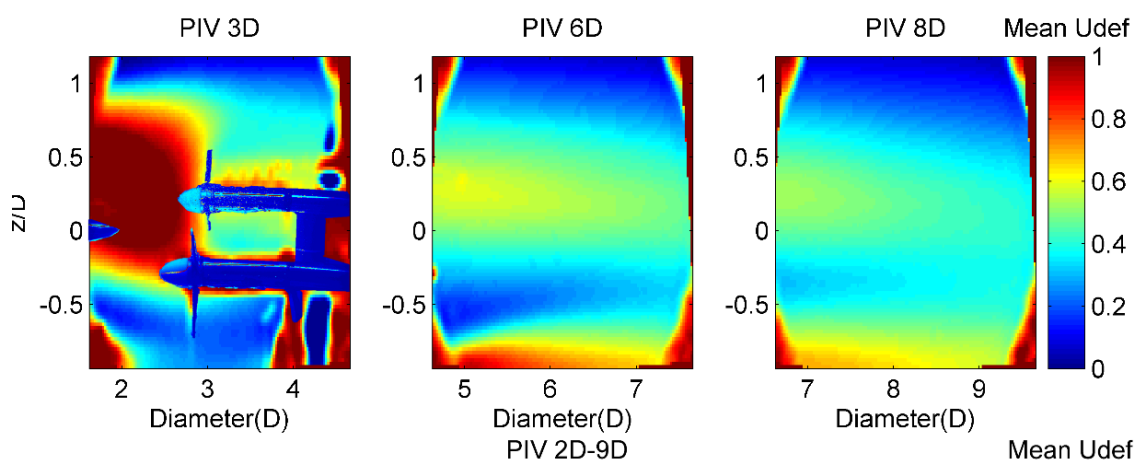

b)

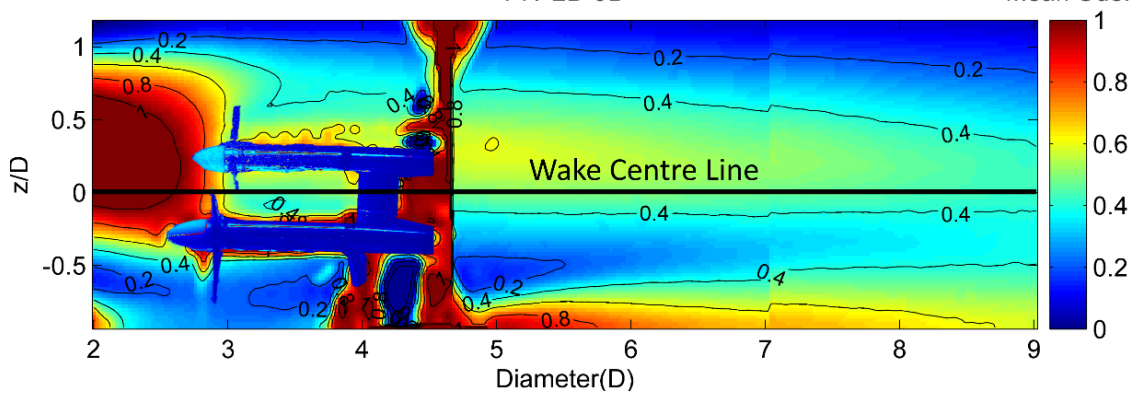

Fig. 19 - (a) Individual and (b) Combined PIV Measurements Including the Position of Second Row Turbines for the Wake in Array L3T15 from 2D - 9D

The resulting wake velocity deficit contours at the centre-line of the array are shown for a number of experiment configurations (Fig. 21 \& Fig. 22). The inner array wake (2D-9D) and the array wake (14D-20D) are shown to visualise areas of increased flow velocity, the wake shape and characteristics as well as effects resulting from the presence of support structure and frame located on the test section floor. Comparison is made for the centre line flow field with longitudinal and transverse variation of the inter-device spacing.

The deficits exceeding unity shown in close proximity are a results of the PIV recording and analysis with very strong velocity gradients where very slow moving flow was observed and reflections of laser light off the turbine hub nacelle. An area of very slow moving fluid directly downstream of the first turbine can be clearly observed with the resulting wake further downstream in Fig. 21, the close longitudinal (L3D) and lateral spacing (a) and is less pronounced for increased transverse spacing (b). Areas of ambient and slightly accelerated flow are identified above and below the wake. For increasing longitudinal spacing in Fig. 22 similar trends of the centre line wake are observed. The wake of the upstream turbine develops further downstream before reaching the second row and an area of reduced velocity deficit is 
1 observed about 1D downstream of the second row rotors. The wake downstream of the arrays

2 show little variation is shown in Section 3.3 however a slight reduction in vertical wake extent

3 can be seen for increased spacing, both longitudinally and transversely.

4 For wide transverse and increased longitudinal spacing in L5T3 in Fig. 22, a more noticeable

5 shift of the wake towards the test section flow is observed than for close longitudinal spacing

6 in Fig. 21 and reduced wake extent is shown up to 20D. This indicates the effect of ambient

7 flow to be stronger, when separation between the first two rows of the array is increased, thus

8 the variations of the onset flow are causing increased mixing at the top of the wake.

9 The support structure wake is most pronounced in the L3T15 array in Fig. 21 a) between 5 and

$109 \mathrm{D}$, indicating that increased blockage area of the three combined rotors at the array test

11 sections diverts the flow around the turbines and increases the presence of the support structure

12 wake.

13 The recovery behind the second row of turbines is shown in Fig. 20 a) comparing the vertical

14 velocity profile at 3D downstream of the second row for L3T15 and L5T3. Wake recovery,

15 especially in the upper part, is accelerated for wide spacing. There is a layer of fluid with

16 increased velocity present in close spacing between the support structure wake and turbine

17 wake, aiding recovery of velocity in the lower half of the wake. The support structure wake is

18 more pronounced in closer spacing as shown in Fig. 20 below -0.5 z/D. Downstream of the

19 array, at 3D and 8D distance from the last turbine, differences between the two formations are

20 reduced, with smaller spacing showing slightly increased deficits at the bottom part of the wake

21 Fig. 20 b). 
a)

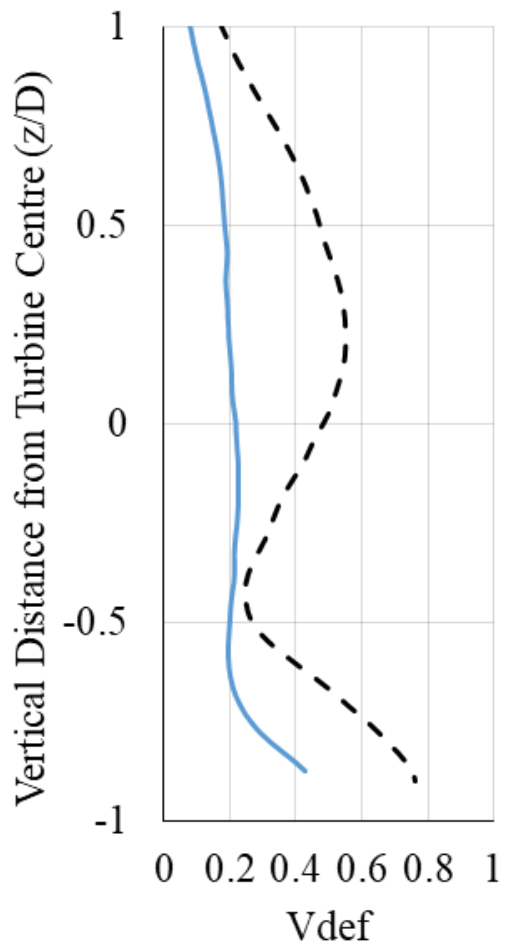

b)

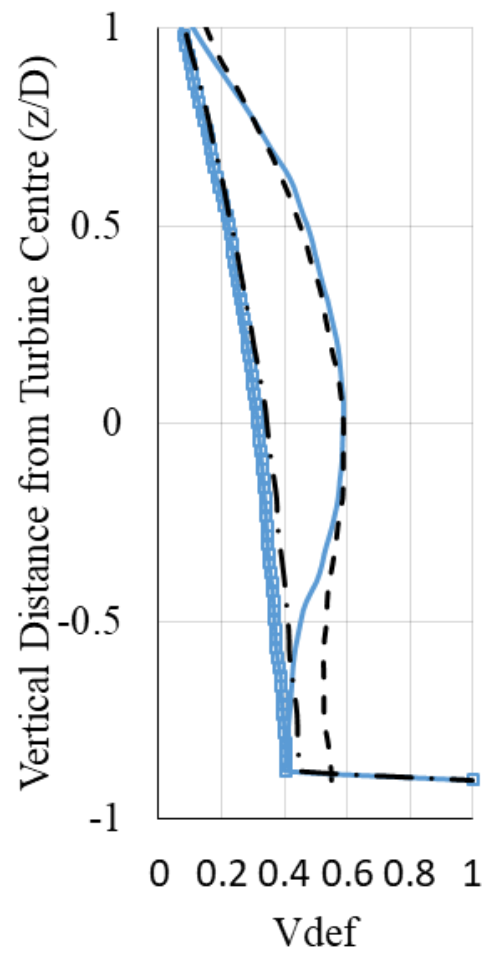

Fig. 20 - Vertical Velocity Profiles at a) 3D downstream of the second row turbines for L3T15 (dash) and L5T3 (solid) respectively and b) 3D (dash L3T15, solid L5T3) as well as 8D (dash dot L3T15, square L5T3) 
a)

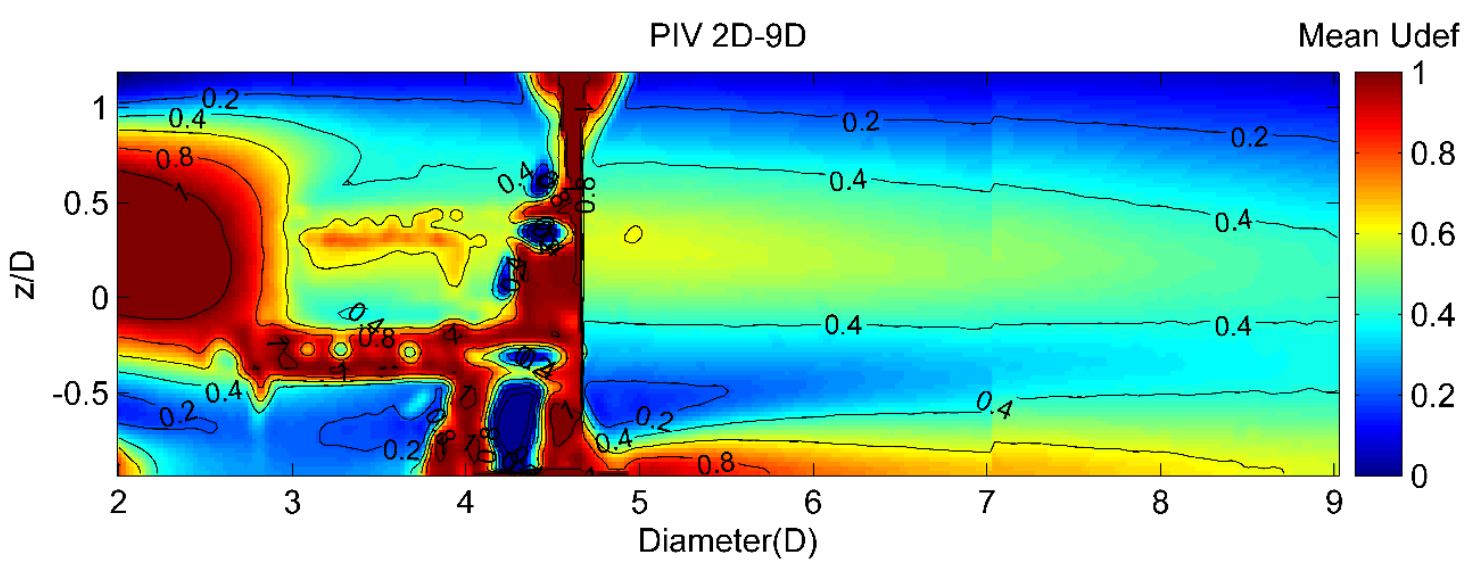

PIV 14D-20D

Mean Udef

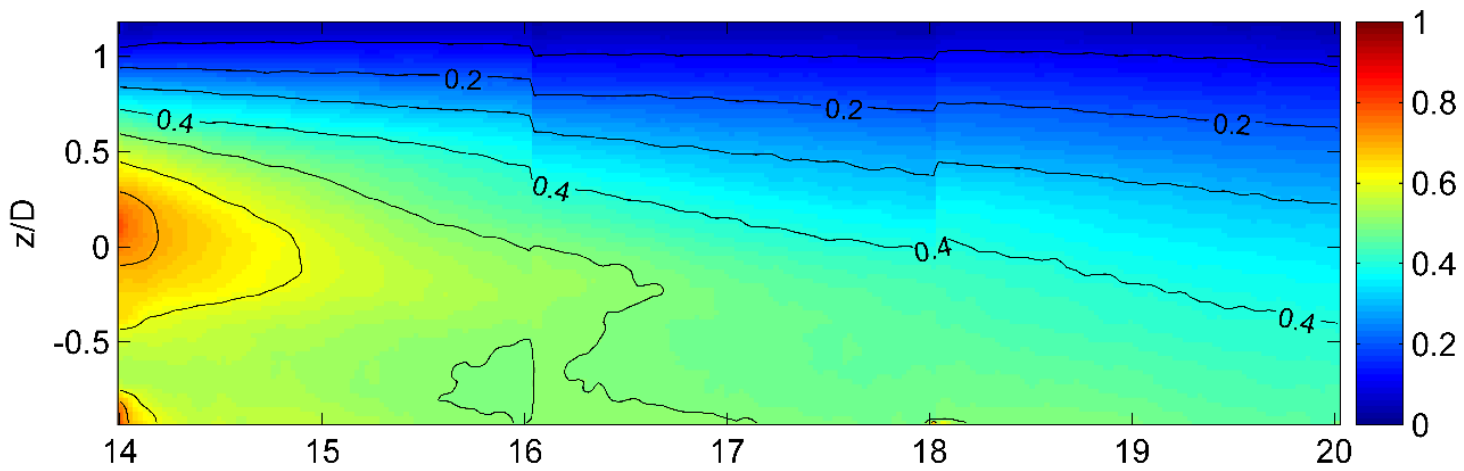

b)
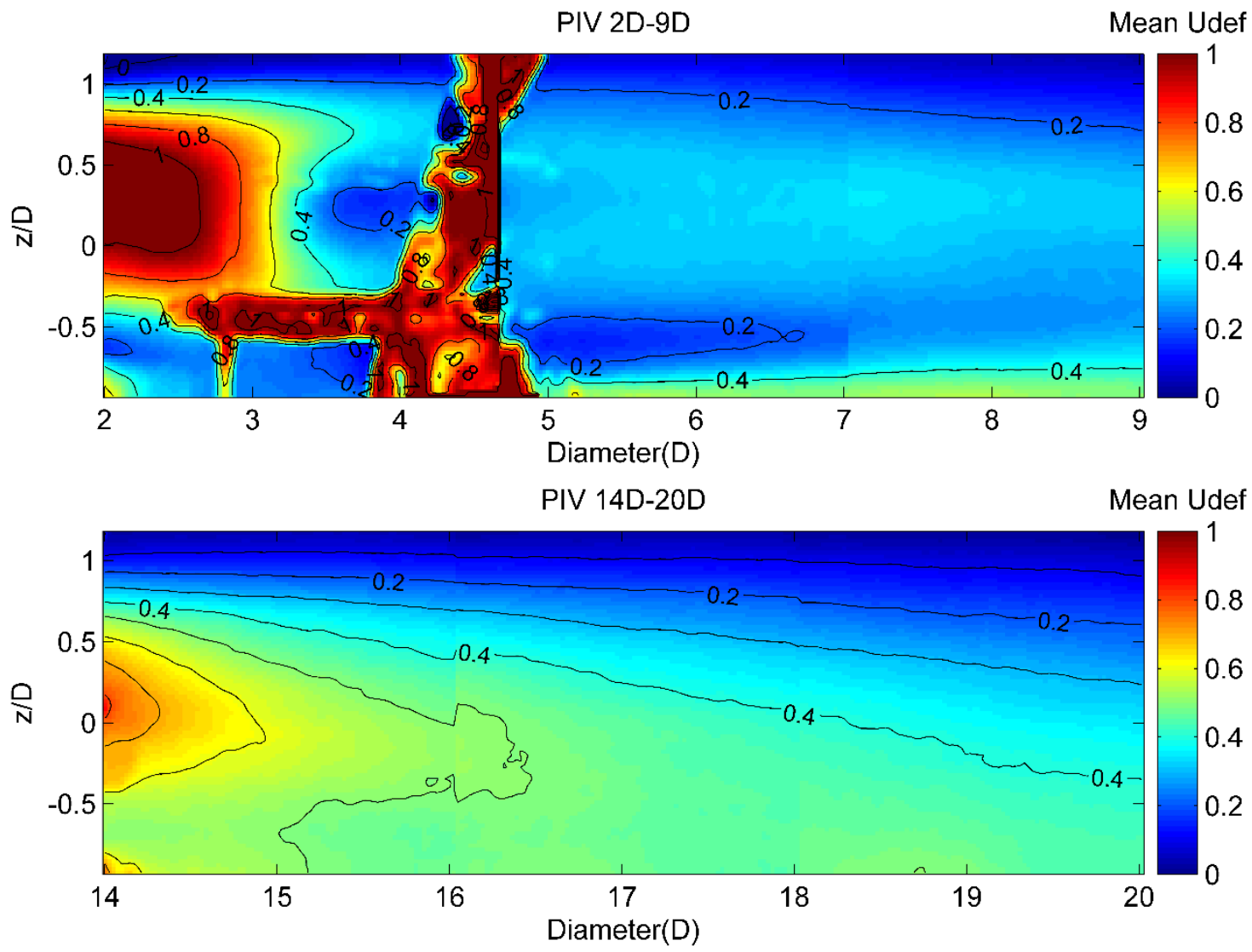

Fig. 21 - Velocity Deficit L3T15 a) and L3T2 b) 
a)
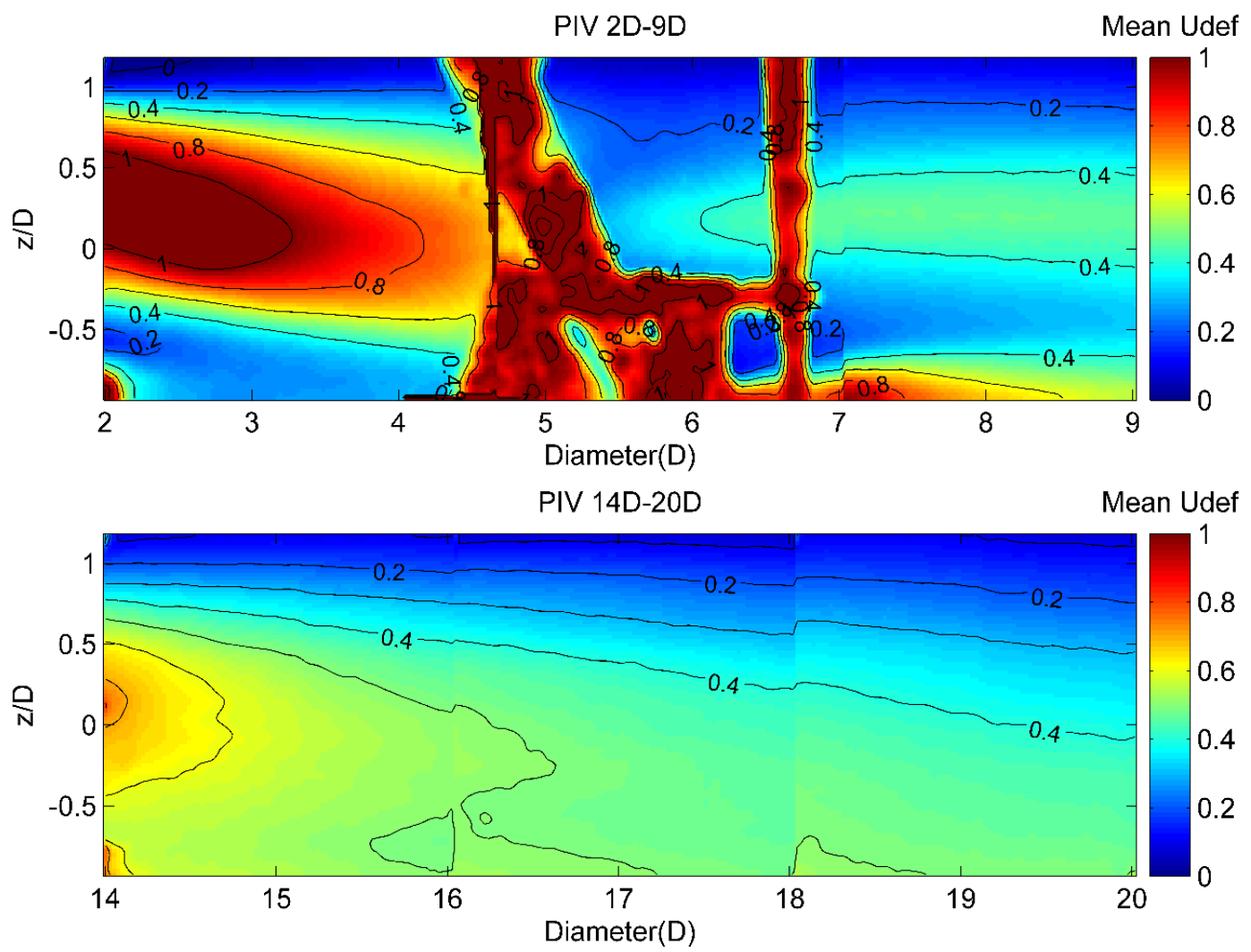

b)
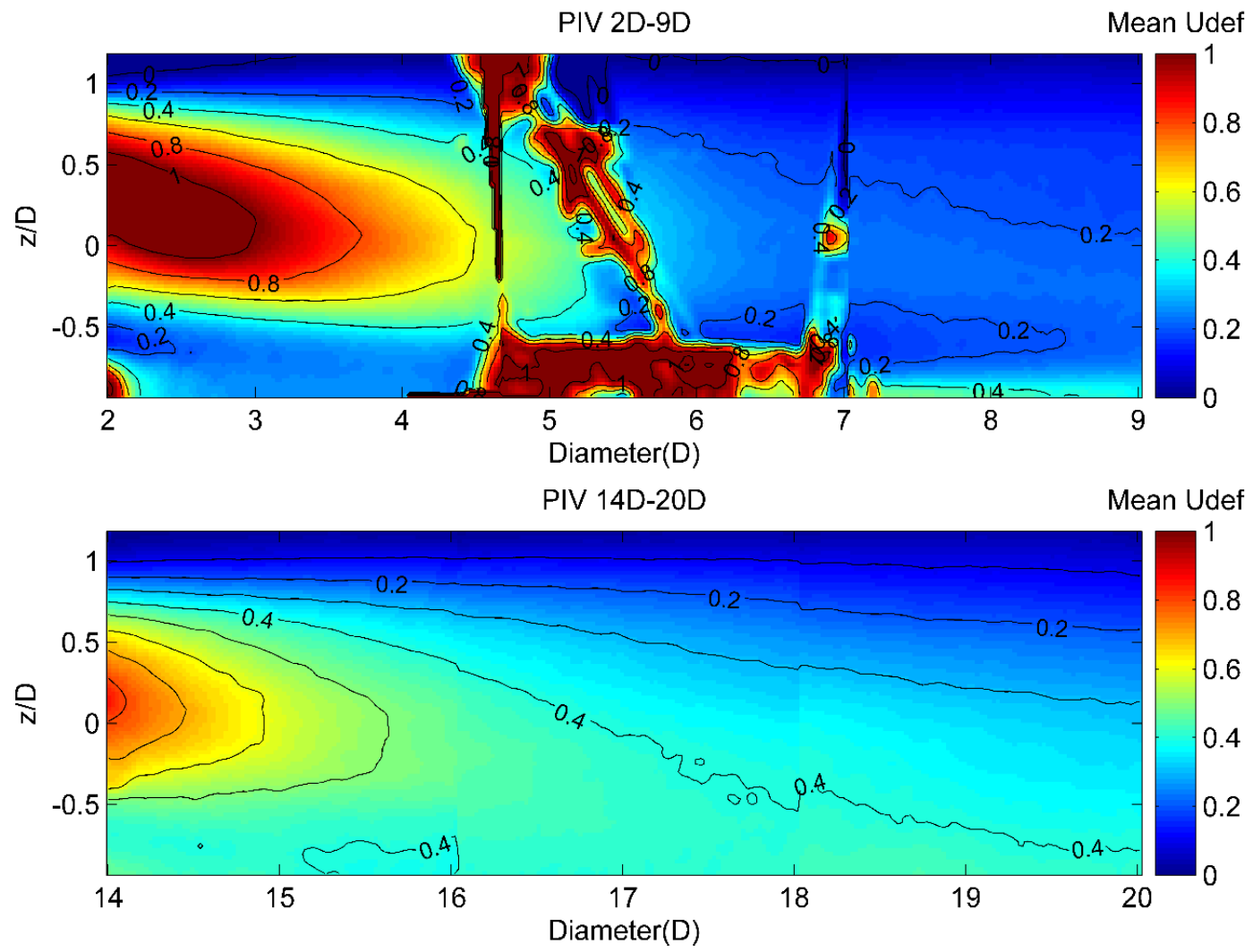

Fig. 22 - Velocity Deficit L5T15 a) and L5T3 b) 


\section{Conclusion}

A comprehensive experimental study has been conducted to assess the influences of turbine spacings within a generic tidal turbine section of a staggered array design layout. Multiple scaled tidal turbine models were designed and operated on a designated support frame utilising a large circulating water channel with PIV measurements along the array centre line to assess the influence of array spacing in lateral and longitudinal direction on the resulting flow field within and downstream of the testsection.

Firstly, a single device has been tested to assess the wake recovery isolated from any other turbines

- Wake velocity and turbulence recovery are strongly linked in this low ambient turbulence setup. A wake velocity deficit of $15 \%$ persists $20 \mathrm{D}$ downstream the isolated device and turbulence levels remain at approximately $8 \%$ compared to the $2 \%$ ambient turbulence levels these finding are comparable to previously studied scaled rotors.

- The wake recovery at the centre-line for a 3 turbine staggered set-up shows that

- Initial wake recovery is sped-up due to more of the ambient flow being channelled around the downstream turbines and towards the array centre-line. Further downstream areas of stagnating and increasing velocity deficit and turbulence intensity have been observed, this is within areas expected to see the expanded wake start merging towards the centre-line wakes.

- The remaining turbulence intensity and wake recovery at $20 \mathrm{D}$ are very similar to that of a single turbine, thus showing the feasibility of a staggered arrangement in terms of velocity recovery prior to locating an additional turbine downstream.

- Multiple array sections were tested to assess the influence of longitudinal and lateral spacing in a three- row array in a staggered 1-2-1 configuration. First and last turbine were located on the array centre line, while the middle row turbines were varied in longitudinal location and transverse spacing. Remaining velocity deficits are within $10 \%$ across all experiments at 20D downstream of the first turbine. Differences in the array wake recovery within the array and 
downstream of the array are attributed to varying lateral and longitudinal spacing and the altered flow field in and around the devices.

- Adding turbines in a staggered arrangement downstream of the first turbine increases initial flow recovery but also shows that very close spacing leads to large velocity deficits at the centre line while wide spacing of the second row shows inflow to the downstream turbine at lower velocity deficits, hence increasing the available power for downstream turbines.

- Array tests have shown some benefits of locating turbines in close lateral spacing by increasing the wake recovery downstream of the array section (L3T15 recovering to lower velocity deficit than L3T2) however would depend on tuning the downstream turbine to the occurring flow as in this experiment the perceived TSR differed from the upstream turbines due to the reduced velocity upstream of the turbine.

- Close spacing increased the effects of the support structure wake visualised by the PIV measurements which led to reduced velocity deficit on the lower part of the wake for the closely spaced arrays, whereas wider spacing saw increased velocity deficits towards the test section floor.

Further testing to characterize lateral wake characteristics will be performed using CFD simulations after validation of numerical model with those of the present experiment. Preliminary simulations conducted in OpenFOAM with fully resolved turbines and dynamic mesh features have been presented [33] and improved modelling is currently being carried out. Further physical measurements and numerical simulation of the lateral wake characterisation are required to confidently identify mixing and wake interaction on an array level. PIV measurements have been applied and shown to provide an alternative flow field measurement for tidal turbine applications and allow for visual investigation of complex flow fields. 


\section{Acknowledgments}

The authors gratefully acknowledge the support received in conducting this experiment by the School of Naval Architecture, Ocean and Civil Engineering at Shanghai Jiao Tong University and the British Council (China) under the "Sino-UK Higher Education Research Partnership" as well as LaVision for providing support and software for the PIV analysis. The numerical work made use of the facilities of N8 HPC provided and funded by the N8 consortium and EPSRC (Grant No.EP/K000225/1). The centre is co-ordinated by the Universities of Leeds and Manchester. 


\section{References}

[1] D.o.E.a.C.C. UK, Renewable Energy Roadmap Update 2013, 2013.

[2] Carbon Trust, Accelerating Marine Energy, 2011.

[3] Atlantis Resources Ltd, NICOLA STURGEON, FIRST MINISTER OF SCOTLAND, UNVEILS WORLD'S LARGEST FREE STREAM TIDAL POWER PROJECT, 2016.

https://atlantisresourcesltd.com/2016/09/13/nicola-sturgeon-first-minister-of-scotland-unveils-worlds-largest-free-stream-tidal-power-project/. (Accessed 8/12/2016 2016).

[4] Atlantis Resources Ltd, MEYGEN UPDATE - FULL POWER GENERATION FROM

TURBINE \#1, 2016. https://atlantisresourcesltd.com/2016/12/07/meygen-update-full-powergeneration-turbine-1/. (Accessed 8/12/2016 2016).

[5] Atlantis Resources Ltd, Projects, 2014. (Accessed 24/08 2014).

[6] Atlantis Resources Ltd, Meygen Project Development \& Operation, 2017.

https://www.atlantisresourcesltd.com/projects/meygen/. (Accessed 31/05/17 2017).

[7] A.S. Bahaj, W.M.J. Batten, G. McCann, Experimental verifications of numerical predictions for the hydrodynamic performance of horizontal axis marine current turbines, Renewable Energy 32(15) (2007) 2479-2490.

[8] W.M. Batten, M.E. Harrison, A.S. Bahaj, Accuracy of the actuator disc-RANS approach for predicting the performance and wake of tidal turbines, Philosophical transactions. Series A,

Mathematical, physical, and engineering sciences 371(1985) (2013) 20120293.

[9] P. Jeffcoate, R. Starzmann, B. Elsaesser, S. Scholl, S. Bischoff, Field measurements of a full scale tidal turbine, International Journal of Marine Energy 12 (2015) 3-20.

[10] S.H. Lee, S.H. Lee, K. Jang, J. Lee, N. Hur, A numerical study for the optimal arrangement of ocean current turbine generators in the ocean current power parks, Current Applied Physics 10(2, Supplement) (2010) S137-S141.

[11] M.J. Churchfield, Y. Li, P.J. Moriarty, A large-eddy simulation study of wake propagation and power production in an array of tidal-current turbines, Philosophical transactions. Series A,

Mathematical, physical, and engineering sciences 371(1985) (2013) 20120421.

[12] R. Ahmadian, R.A. Falconer, Assessment of array shape of tidal stream turbines on hydroenvironmental impacts and power output, Renewable Energy 44 (2012) 318-327.

[13] S.W. Funke, P.E. Farrell, M.D. Piggott, Tidal turbine array optimisation using the adjoint approach, Renewable Energy 63 (2014) 658-673.

[14] C. Garrett, P. Cummins, The efficiency of a turbine in a tidal channel, Journal of Fluid

Mechanics 588 (2007) 243-251.

[15] T. Nishino, R.H.J. Willden, The efficiency of an array of tidal turbines partially blocking a wide channel, Journal of Fluid Mechanics 708 (2012) 596-606.

[16] S. Draper, T. Nishino, Centred and staggered arrangements of tidal turbines, Journal of Fluid Mechanics 739 (2013) 72-93.

[17] P. Mycek, B. Gaurier, G. Germain, G. Pinon, E. Rivoalen, Experimental study of the turbulence intensity effects on marine current turbines behaviour. Part I: One single turbine, Renewable Energy 66(0) (2014) 729-746.

[18] S.C. Tedds, I. Owen, R.J. Poole, Near-wake characteristics of a model horizontal axis tidal stream turbine, Renewable Energy 63(0) (2014) 222-235.

[19] L.P. Chamorro, D.R. Troolin, S.J. Lee, R.E.A. Arndt, F. Sotiropoulos, Three-dimensional flow visualization in the wake of a miniature axial-flow hydrokinetic turbine, Exp Fluids 54(2) (2013) 112.

[20] P. Mycek, B. Gaurier, G. Germain, G. Pinon, E. Rivoalen, Experimental study of the turbulence intensity effects on marine current turbines behaviour. Part II: Two interacting turbines, Renewable Energy 68 (2014) 876-892.

[21] L.E. Myers, A.S. Bahaj, An experimental investigation simulating flow effects in first generation marine current energy converter arrays, Renewable Energy 37(1) (2012) 28-36. 
[22] T. Stallard, R. Collings, T. Feng, J. Whelan, Interactions between tidal turbine wakes: experimental study of a group of three-bladed rotors, Philosophical transactions. Series A, Mathematical, physical, and engineering sciences 371(1985) (2013) 20120159.

[23] A. Olczak, T. Stallard, T. Feng, P.K. Stansby, Comparison of a RANS blade element model for tidal turbine arrays with laboratory scale measurements of wake velocity and rotor thrust, Journal of Fluids and Structures 64 (2016) 87-106.

[24] W. Shi, M. Atlar, R. Norman, Detailed flow measurement of the field around tidal turbines with and without biomimetic leading-edge tubercles, Renewable Energy 111 (2017) 688-707.

[25] A. Good, G. Hamill, T. Whittaker, D. Robinson, PIV analysis of the near wake of a tidal turbine, Proc. ISOPE, Maui, Hawaii, 2011.

[26] L.E. Myers, A.S. Bahaj, Experimental analysis of the flow field around horizontal axis tidal turbines by use of scale mesh disk rotor simulators, Ocean Engineering 37(2-3) (2010) 218-227. [27] W.C. Shi, D. Wang, M. Atlar, K.C. Seo, Flow separation impacts on the hydrodynamic performance analysis of a marine current turbine using CFD, P I Mech Eng a-J Pow 227(8) (2013) 833-846.

[28] N.K. Stelzenmuller, Marine Hydrokinetic Turbine Array Performance and Wake Characteristics, University of Washington, 2013.

[29] L.P. Chamorro, R.E.A. Arndt, F. Sotiropoulos, Reynolds number dependence of turbulence statistics in the wake of wind turbines, Wind Energy 15(5) (2012) 733-742.

[30] A. Mason-Jones, D.M. O'Doherty, C.E. Morris, T. O'Doherty, C.B. Byrne, P.W. Prickett, R.I. Grosvenor, I. Owen, S. Tedds, R.J. Poole, Non-dimensional scaling of tidal stream turbines, Energy 44(1) (2012) 820-829.

[31] D.A. Doman, R.E. Murray, M.J. Pegg, K. Gracie, C.M. Johnstone, T. Nevalainen, Tow-tank testing of a 1/20th scale horizontal axis tidal turbine with uncertainty analysis, International Journal of Marine Energy 11 (2015) 105-119.

[32] F. Maganga, G. Germain, J. King, G. Pinon, E. Rivoalen, Experimental characterisation of flow effects on marine current turbine behaviour and on its wake properties, Iet Renewable Power Generation 4(6) (2010) 498-509.

[33] M. Nuernberg, L. Tao, Experimental Study of Flow Field Characteristics in Tidal Stream Turbine Arrays, Proceedings of the International Conference on Offshore Mechanics and Arctic Engineering - OMAE (49972) (2016) V006T09A004. 